\title{
Flow-Velocity and Depth Data During Peak Discharge Events at Selected Bridge Crossings in North Carolina, 1964-98
}

By Benjamin F. Pope

U.S. GEOLOGICAL SURVEY

Open-File Report 00-169

Prepared in cooperation with the

North Carolina Department of Transportation

Raleigh, North Carolina 2000

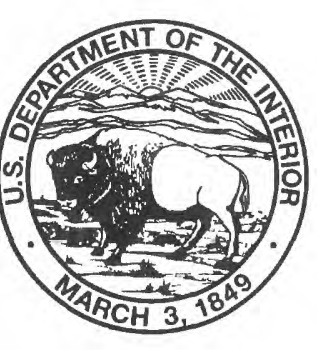




\title{
U.S. DEPARTMENT OF THE INTERIOR BRUCE BABBITT, Secretary
}

\author{
U.S. GEOLOGICAL SURVEY
}

Charles G. Groat, Director

The use of firm, trade, and brand names in this report is for identification purposes only and does not constitute endorsement by the U.S. Geological Survey.

For additional information write to:

District Chief

U.S. Geological Survey 3916 Sunset Ridge Road

Raleigh, NC 27607-6416
Copies of this report can be purchased from:

U.S. Geological Survey Branch of Information Services Box 25286, Denver Federal Center Denver, CO 80225 


\section{CONTENTS}

Abstract

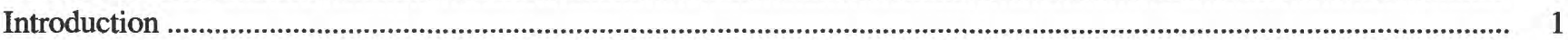

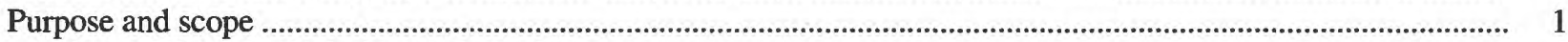

Flow velocity and flow depth during peak discharges.......................................................................................................... 4

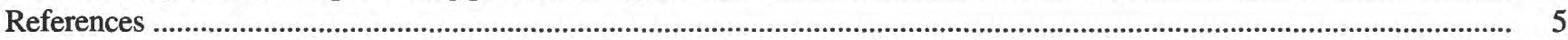

\section{FIGURES}

1. Locations of selected streamgaging stations in North Carolina ........................................................................ 2

2-22. Channel cross sections and maximum flow velocities at:

2. Station 02053200, Potecasi Creek near Union, N.C. .......................................................................... 6

3. Station 02074000, Smith River at Eden, N.C. ........................................................................................... 8

4. Station 02083500, Tar River at Tarboro, N.C. ….................................................................................. 10

5. Station 02090380, Contentnea Creek near Lucama, N.C. ............................................................................. 12

6. Station 02093000, New River near Gum Branch, N.C. ....................................................................... 14

7. Station 02096500, Haw River at Haw River, N.C. .................................................................................. 16

8. Station 02104220, Rockfish Creek at Raeford, N.C. ..................................................................................... 18

9. Station 02112250, Yadkin River at Elkin, N.C. .................................................................................. 20

10. Station 02113000, Fisher River near Copeland, N.C. ................................................................................ 22

11. Station 02129000 , Pee Dee River near Rockingham, N.C. ................................................................. 24

12. Station 02133500 , Drowning Creek near Hoffman, N.C. ........................................................................... 26

13. Station 02133624, Lumber River near Maxton, N.C. .............................................................................. 28

14. Station 02137727, Catawba River near Pleasant Gardens, N.C. ............................................................. 30

15. Station 02143000 , Henry Fork near Henry River, N.C. ............................................................................... 32

16. Station 02146900, Twelve Mile Creek near Waxhaw, N.C. .................................................................... 34

17. Station 02149000, Cove Creek near Lake Lure, N.C. ......................................................................... 36

18. Station 03439000, French Broad River at Rosman, N.C. ............................................................................ 38

19. Station 03443000, French Broad River at Blantyre, N.C. ........................................................................... 40

20. Station 03451500, French Broad River at Asheville, N.C. ................................................................. 42

21. Station 03463300, South Toe River near Celo, N.C. ............................................................................... 44

22. Station 03500240, Cartoogechaye Creek near Franklin, N.C. .............................................................. 46

CONVERSION FACTORS and VERTICAL DATUM

\begin{tabular}{rcl}
\hline Multiply & by & To obtaln \\
\hline & Length & \\
foot $(\mathrm{ft})$ & 0.3048 & meter \\
mile $(\mathrm{mi})$ & 1.609 & kilometer \\
& Area & \\
square mile $\left(\mathrm{mi}^{2}\right)$ & 2.590 & square kilometer \\
& Flow & \\
foot per second $(\mathrm{ft} / \mathrm{s})$ & 0.3048 & meter per second \\
cubic foot per second $\left(\mathrm{ft}^{3} / \mathrm{s}\right)$ & 0.02832 & cubic meter per second \\
\hline
\end{tabular}

Sea level: In this report, "sea level" refers to the National Geodetic Vertical Datum of 1929 (NGVD of 1929), a geodetic datum derived from a general adjustment of the first-order level nets of both the United States and Canada, formerly called Sea Level Datum of 1929. 


\title{
Flow-Velocity and Depth Data During Peak Discharge Events at Selected Bridge Crossings in North Carolina, 1964-98
}

\author{
By Benjamin F. Pope
}

\section{ABSTRACT}

Flow-velocity and depth data were collected from July 1996 through December 1998 during peak discharge events at 21 bridge crossings that are adjacent to U.S. Geological Survey streamgaging stations in North Carolina. These data were collected during measurements of peak discharges that had recurrence intervals ranging from less than 2 years to about 100 years. The velocity and depth data can be used to evaluate predicted flow velocities and scour depths that are computed as part of scour analyses at the selected bridge crossings.

\section{INTRODUCTION}

River and stream channels are dynamic, changing in width and depth, or both, over time. These changes are the result of complex, interrelated natural processes. Bridge crossings of river or stream channels can alter the dynamic natural processes in the channels by (1) constricting the cross-sectional area of streamflow during high flows, (2) reducing the amount of streambed and streambank area that is available to supply sediment, and (3) acting as obstructions that redirect flow. Erosion of the channel bottom or banks at bridge crossings generally is referred to as bridge scour. Bridge scour can undermine bridge abutments, foundations, and pier footings. Estimates of potential bridge scour are used to design new bridges and assess the safety of existing bridges.

Scour depths at bridges are predicted by using empirically based equations; an important variable in these equations is flow velocity. Flow velocities used in scour prediction equations typically are determined by applying one- or two-dimensional steady-flow numerical models of the stream reach upstream and downstream from the bridge crossing to a specified discharge. Errors in flow velocities that are predicted by these numerical models may result in predicted scour depths that differ from actual scour. Predicted scour also may differ from actual scour because of the dynamic nature of the flow at bridges and differences between scale model and prototype conditions. Actual velocities and depths of flow, measured during peak discharge events, can be compared to predicted flow velocities and predicted scour depths in order to evaluate the accuracy of the predicted values.

\section{Purpose and Scope}

In July 1996 the U.S. Geological Survey (USGS) and the North Carolina Department of Transportation (NCDOT) began a cooperative study to (1) collect flow-velocity and flow-depth data during peak discharge events at selected bridge crossings at or near USGS streamgaging stations in North Carolina, and (2) review historic measurements of peak discharge at these bridge crossings and extract the relevant flowvelocity and flow-depth data.

This report presents flow-velocity and depth data collected during measurements of peak discharges that were made at 21 USGS streamgages across the State from September 1964 through December 1998 (fig. 1). Data from measurements of discharges with a recurrence interval greater than about 1.5 years that were made at the selected sites during the active datacollection period from July 1996 through December 1998 are included in this report. In addition to measurements made during the data-collection period, 


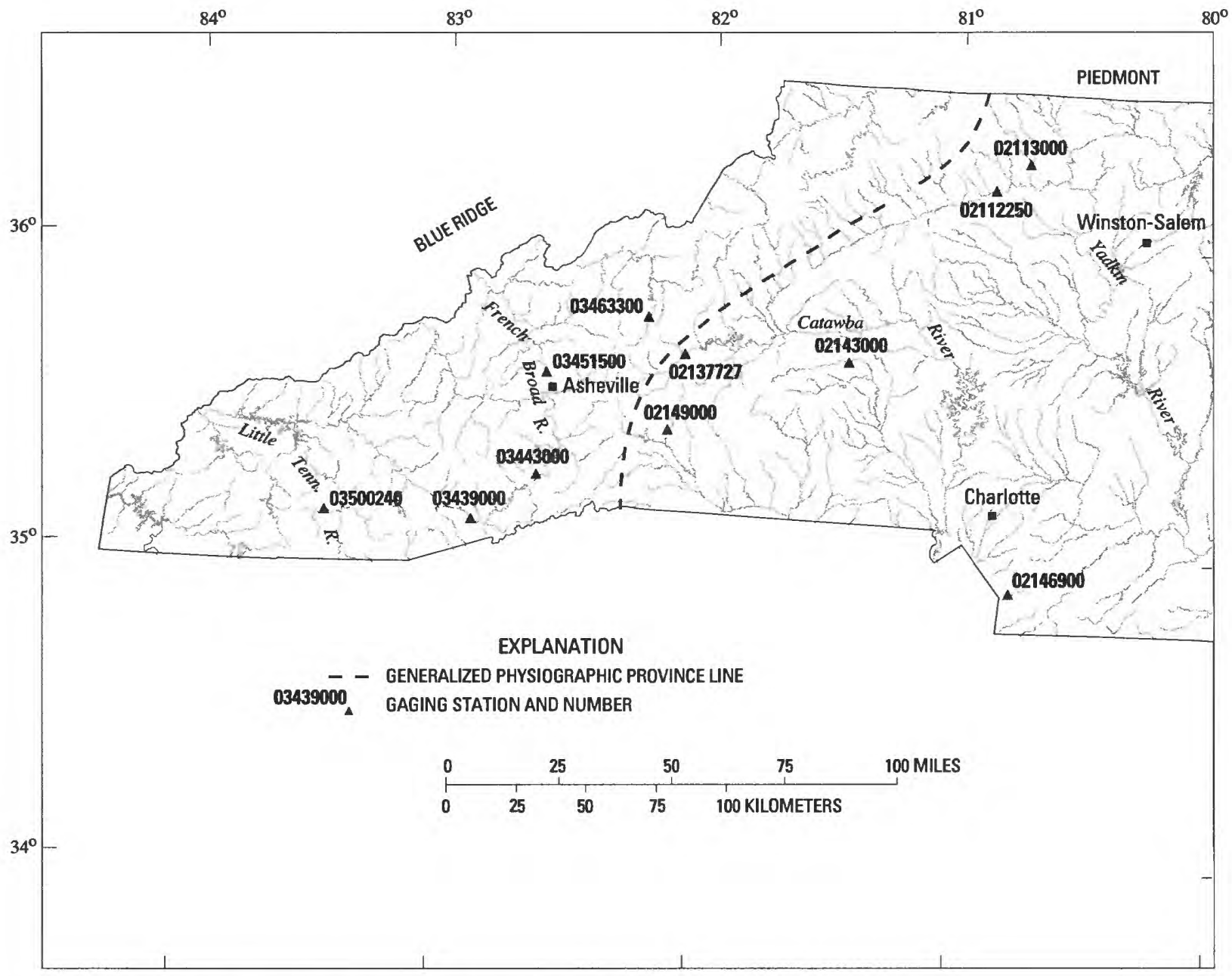

Figure 1. Locations of selected streamgaging stations in North Carolina. 


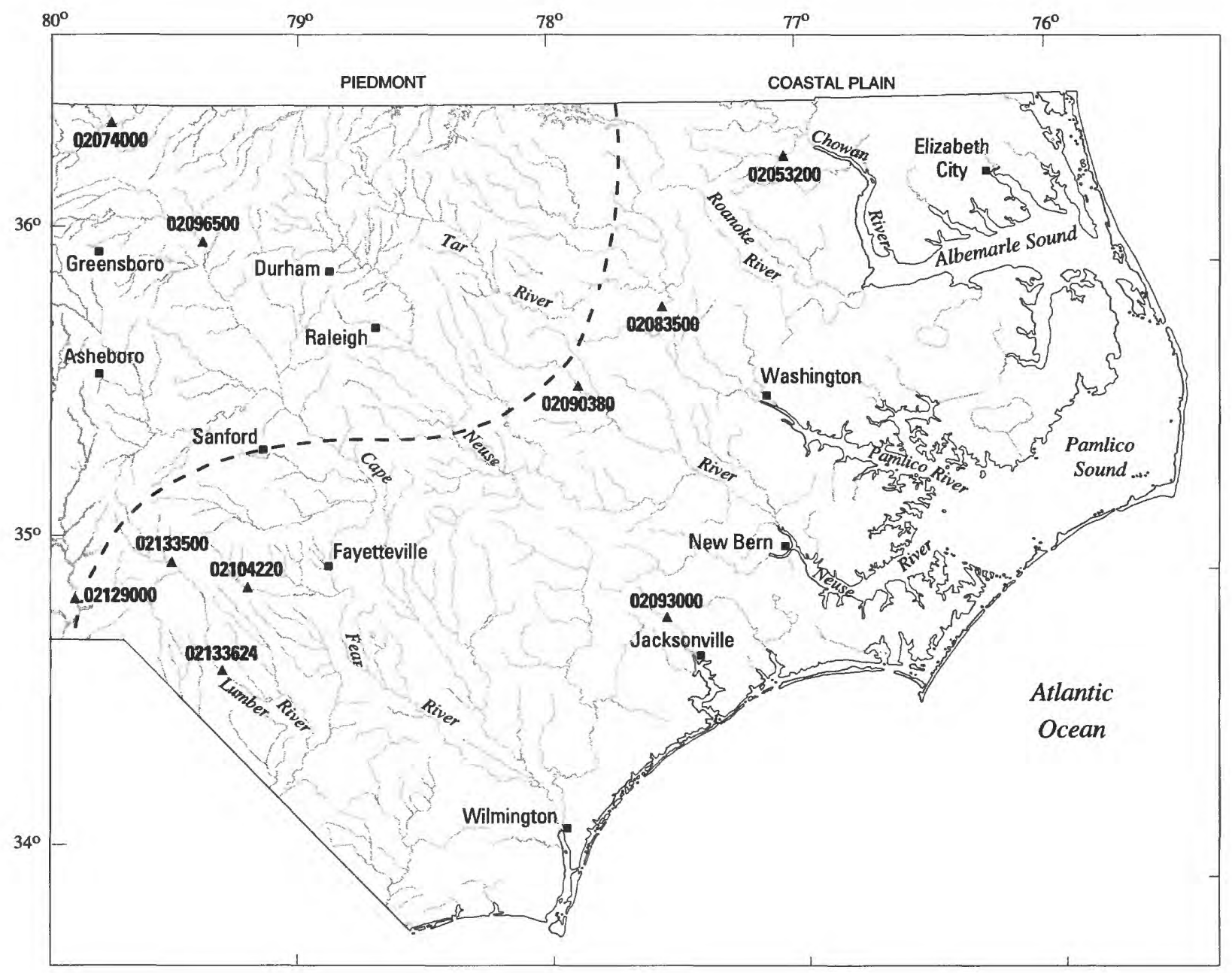


discharge measurements made prior to July 1996 at each site were reviewed and the largest one or two discharge measurements with a recurrence interval greater than 1.5 years were noted. Measurements were then selected for inclusion in this report so that (1) the largest measurement made at a site, regardless of period, was included, (2) the largest measurement made during the active data-collection period was included, and (3) the more recent discharge measurement was included if more than one measurement had been made at approximately equal discharges. At several of the selected sites no discharge measurements with a recurrence interval greater than 1.5 years were made during the data-collection period. For these sites, only data from measurements made prior to July 1996 are included.

\section{FLOW VELOCITY AND FLOW DEPTH DURING PEAK DISCHARGES}

In coordination with the NCDOT, 25 USGS streamgaging sites in North Carolina where peak discharge measurements are typically made at adjacent bridge crossings were initially selected as potential sites for the collection of flow-velocity and depth data. Four of the initial 25 sites, however, were identified later as being unsuitable for the study. Because of difficult measurement conditions at these four sites during large discharge events, indirect methods of discharge computation were used to determine large peak discharges; measured velocity and flow data during peak discharges are not available. As a result, these four sites were deleted from the study, leaving a total of 21 study sites.

Discharge measurements are routinely made throughout the range of observed stream levels, or stages, at USGS streamgages in order to define the relation between stage and discharge. Bridge scour, however, is associated with the high flow velocities that occur during large discharge events. For this reason, data from measurements made during large discharge events are presented in this report. Because the magnitude of a large discharge event at a given streamgage is relative to the area drained by the stream at the gage location, the recurrence interval of the discharge event was used as a criterion for (1) measuring the discharge at a selected streamgaging site during the data-collection period of the study, and (2) inclusion in this report for discharge measurements made prior to the data-collection period. Recurrence interval of a discharge is defined as the number of years, on average, during which a specific discharge is expected to be equaled or exceeded one time. For instance a 2-year discharge is expected to be equaled or exceeded, on average, once every 2 years. Estimates of recurrence intervals at streamgaging sites are computed by fitting the highest peak for each year of gage record to a statistical distribution. At least 10 years of discharge data are required to compute recurrence interval discharges at a given streamgage. Recurrence interval discharges for the streamgaging sites in North Carolina, including those used in this report, as well as a more detailed description of the computations are provided by Pope and Tasker (1999).

Discharge measurements that had a recurrence interval of at least 1.5 years were made at 9 of the 21 sites during the data-collection period, July 1996 through December 1998. Flow-velocity and flowdepth data from those measurements are presented in this report. At 12 of the 21 sites, no discharge measurements with a recurrence interval of at least 1.5 years were made during the data-collection period. Data from measurements made prior to July 1996 are presented for these 12 sites. At four of the sites where discharge measurements were made during the data-collection period, significantly larger discharges were measured prior to July 1996. At these four sites, data from discharge measurements that were made prior to the data-collection period are presented along with data from discharge measurements made during the data-collection period.

The flow-velocity and flow-depth data presented in this report were collected while measuring discharge during high flow conditions at the selected sites. Discharge is typically measured by establishing a horizontal cross section, roughly perpendicular to the direction of flow, and making measurements of flow depth and velocity at several points, or vertical sections, along the cross section. A lead weight is used to make depth soundings, or measurements, at each vertical section. Flow velocities are measured by using a current meter at several depths at each vertical section in the cross section. Velocities are typically measured either at a single depth, 0.6 of the total depth, or at two depths, 0.2 and 0.8 of the total depth. Velocity measured at the 0.6 depth is assumed to be the average velocity for that vertical section, and the average of the velocities measured at the 0.2 and 0.8 depths is assumed to be the average velocity for that vertical section. The width between vertical sections and the 
depth of flow at each vertical section are used to compute the flow area at that vertical section. A volumetric rate of flow, or discharge, for each vertical section is computed by multiplying the average flow velocity at each section by the flow area for that section. Total stream discharge is the sum of the discharges computed at each section. A more detailed description of the techniques and equipment used in measuring discharge is provided by Rantz and others (1982). In some instances, additional depth soundings were made to further define the channel cross section during discharge measurements made during the datacollection period of this study.

Data provided for each site consist of a station summary, a discharge measurement summary, a figure showing the channel cross section and measured velocities for each discharge measurement presented, and a table of the flow-velocity and flow-depth data for each discharge measurement. The station summary provides descriptive information about each of the study sites, including gage location, location of measurement, period of record, elevation of the gage datum, and any extreme events that occurred outside the period of record. The discharge measurement summary includes descriptive information about the discharge measurements from which the depth and velocity data were extracted. The figure presents depth-of-flow data by showing an outline of the channel bottom, as defined by depth soundings made during the measurement. The velocity data are represented by a series of shaded bars. The position of a bar along the horizontal axis represents the location of the vertical section, referenced to an arbitrary starting point located on the left bank of the stream, at which depth and velocity were measured. The height of the bar represents the magnitude of the maximum velocity measured at that point. For each discharge measurement presented, a data table including location of each vertical section, depth from water surface to channel bottom at each section, and maximum velocity measured at each section also is provided.

\section{REFERENCES}

Pope, B.F., and Tasker, G.D., 1999, Estimating the magnitude and frequency of floods in rural basins of North Carolina: U.S. Geological Survey WaterResources Investigations Report 99-4114, 44 p. + erratum.

Rantz, S.E., and others, 1982, Measurement and computation of streamflow: Volume 1. Measurement of stage and discharge: U.S. Geological Survey WaterSupply Paper 2175, p. 79-183. 


\section{POTECASI CREEK NEAR UNION, N.C.}

GAGE LOCATION.--Lat $36^{\circ} 22^{\prime} 14^{\prime \prime}$, long $77^{\circ} 01^{\prime} 36^{\prime \prime}$, Hertford County, Hydrologic Unit 03010204 , on right bank at downstream side of bridge on State Highway 11, 2.8 mi north of Union, 3 mi downstream of Cutawhiskie Swamp, and 3.5 mi upstream from Bells Branch.

MEASUREMENT LOCATION.--Upstream side of bridge on State Highway 11.

DRAINAGE AREA.--225 $\mathrm{mi}^{2}$.

PERIOD OF RECORD.--Occasional low-flow measurements, water years 1953-57. March 1958 to current year.

GAGE.--Water-stage recorder with satellite telemetry. Datum of gage is $3.53 \mathrm{ft}$ above sea level. Prior to Dec. 1, 1958, nonrecording gage at same site and datum.

EXTREMES OUTSIDE PERIOD OF RECORD.--Flood in 1929 reached a stage of $19.1 \mathrm{ft}$; discharge, $4,050 \mathrm{ft} / \mathrm{s}$; and flood of August 1940 reached a stage of $24.1 \mathrm{ft}$; discharge, $7,000 \mathrm{ft}^{3} / \mathrm{s}$, from rating curve extended above $4,000 \mathrm{ft} 3 / \mathrm{s}$, from information furnished by North Carolina State Highway Commission.

\section{DISCHARGE MEASUREMENT SUMMARY}

[ft/s, foot per second; $\mathrm{ft}$, foot; $\mathrm{ft}^{3} / \mathrm{s}$, cubic foot per second]

\begin{tabular}{ccccccc}
\hline $\begin{array}{c}\text { Date } \\
\text { of } \\
\text { measurement }\end{array}$ & $\begin{array}{c}\text { Stream stage } \\
\text { (feet above } \\
\text { datum) }\end{array}$ & $\begin{array}{c}\text { Average } \\
\text { velocity } \\
(\mathrm{ft} / \mathrm{s})\end{array}$ & $\begin{array}{c}\text { Maximum } \\
\text { velocity } \\
(\mathrm{ft} / \mathrm{s})\end{array}$ & $\begin{array}{c}\text { Maximum } \\
\text { depth } \\
(\mathrm{ft})\end{array}$ & $\begin{array}{c}\text { Discharge } \\
\left(\mathrm{ft}^{3} / \mathrm{s}\right)\end{array}$ & $\begin{array}{c}\text { Recurrence } \\
\text { interval } \\
(\text { years) }\end{array}$ \\
\hline $08-18-92$ & 21.54 & 1.93 & 3.13 & 22.5 & 5,550 & 50 \\
$08-21-92$ & 18.31 & 1.43 & 2.74 & 19.1 & 3,240 & $5-10$ \\
\hline
\end{tabular}

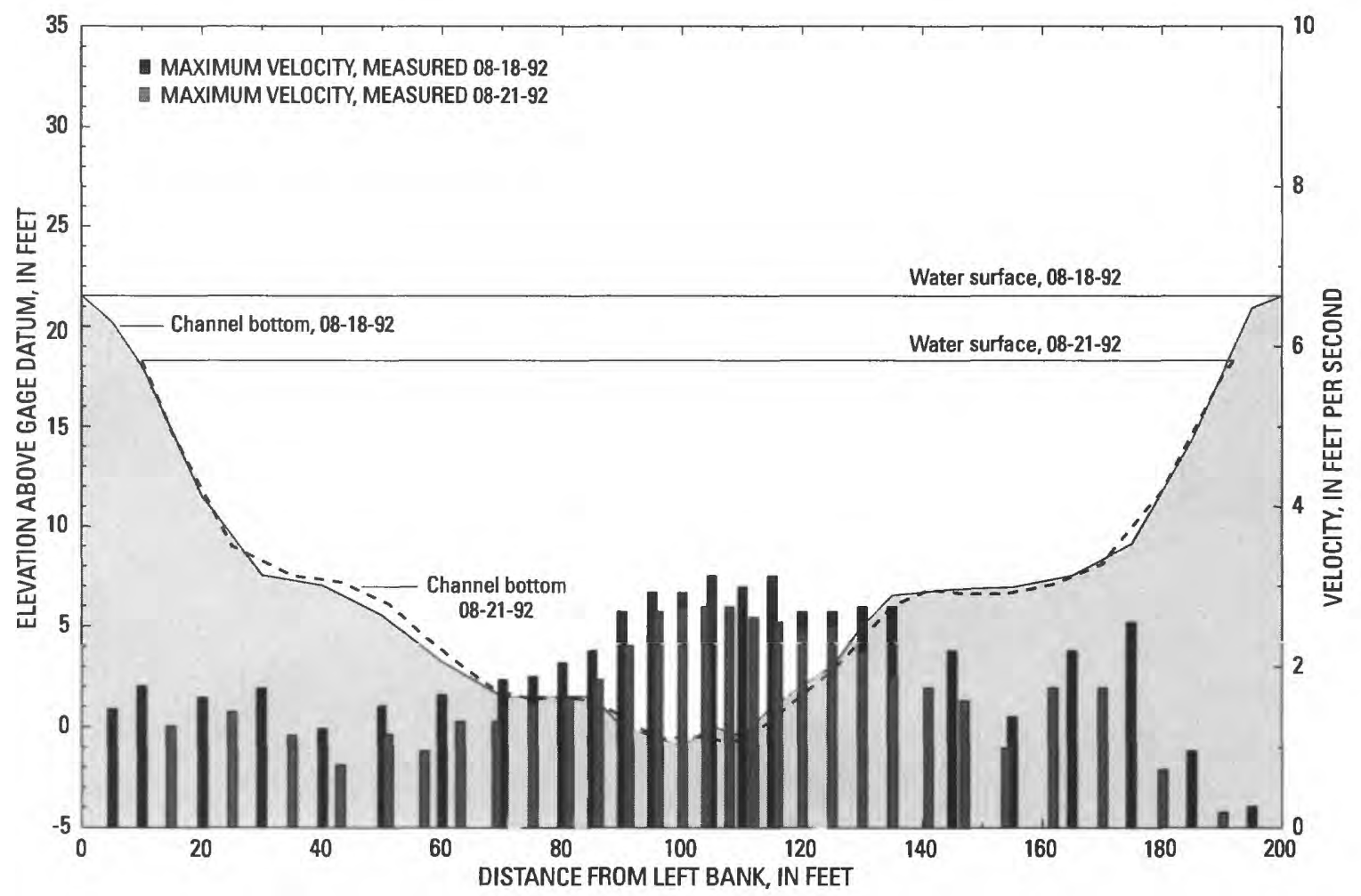

Figure 2. Channel cross sections and maximum flow velocities at station 02053200 Potecasi Creek near Union, N.C. 
DISCHARGE MEASUREMENT DATA FOR STATION 02053200 POTECASI CREEK NEAR UNION, N.C.

[ft, foot; ft/s, foot per second; nd, no data]

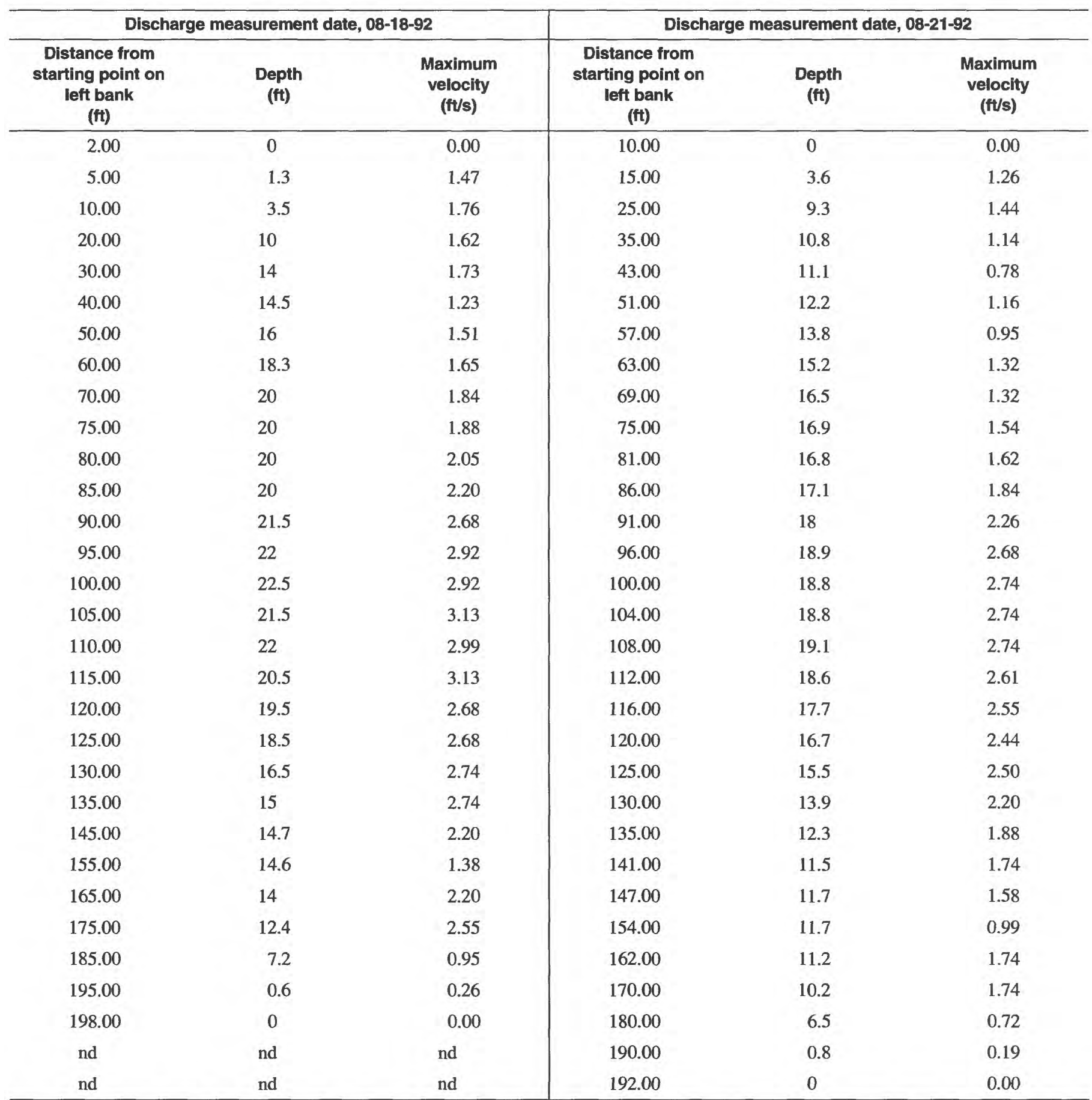




\section{SMITH RIVER AT EDEN, N.C.}

GAGE LOCATION.--Lat 36 31'31", long 7945'57", Rockingham County, Hydrologic Unit 03010103, on right bank at Eden, 0.3 mi downstream of bridge on State Highway 14, 0.8 mi upstream from bridge on Secondary Road 1714, 1.2 mi south of Virginia-North Carolina State line, 1.3 mi downstream of Stuart Creek, and 3.9 mi upstream from mouth.

MEASUREMENT LOCATION.--Downstream side of bridge on State Highway 14.

DRAINAGE AREA.--538 $\mathrm{mi}^{2}$.

PERIOD OF RECORD.--October 1939 to current year. Prior to October 1970, published as "Smith River at Spray."

GAGE.--Water-stage recorder with satellite telemetry. Datum of gage is $539.56 \mathrm{ft}$ above sea level.

\section{DISCHARGE MEASUREMENT SUMMARY}

[ft/s, foot per second; $\mathrm{ft}$, foot; $\mathrm{ft}^{3} / \mathrm{s}$, cubic foot per second]

\begin{tabular}{ccccccc}
\hline $\begin{array}{c}\text { Date } \\
\text { of } \\
\text { measurement }\end{array}$ & $\begin{array}{c}\text { Stream stage } \\
\text { (feet above } \\
\text { datum) }\end{array}$ & $\begin{array}{c}\text { Average } \\
\text { velocity } \\
(\mathrm{ft} / \mathrm{s})\end{array}$ & $\begin{array}{c}\text { Maximum } \\
\text { velocity } \\
(\mathrm{ft} / \mathrm{s})\end{array}$ & $\begin{array}{c}\text { Maximum } \\
\text { depth } \\
(\mathrm{ft})\end{array}$ & $\begin{array}{c}\text { Discharge } \\
\left(\mathrm{ft}^{3} / \mathrm{s}\right)\end{array}$ & $\begin{array}{c}\text { Recurrence } \\
\text { interval } \\
(\text { (years) }\end{array}$ \\
\hline $06-21-72$ & 16.20 & 4.85 & 8.19 & 25.2 & 24,500 & 25 \\
\hline
\end{tabular}

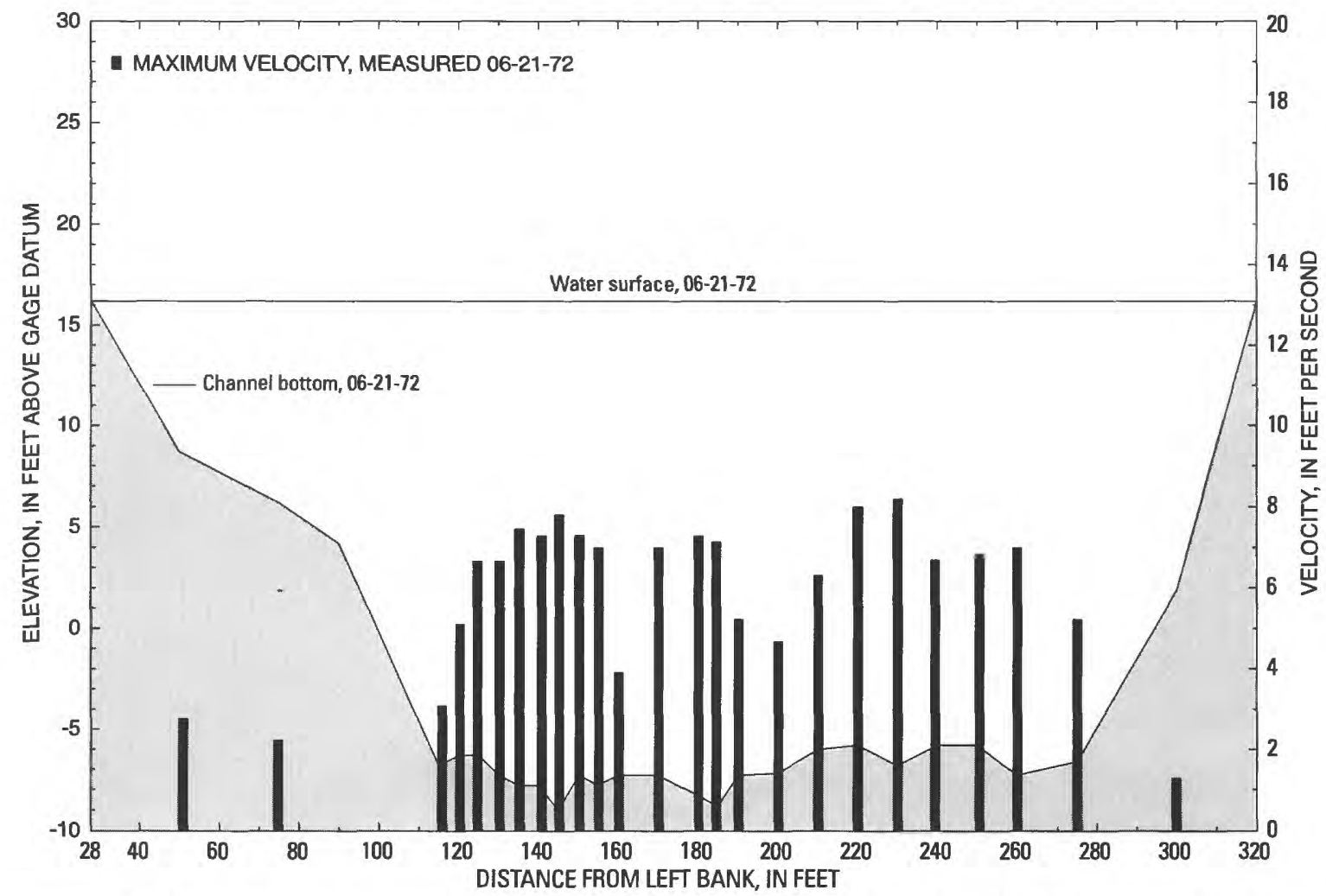

Figure 3. Channel cross section and maximum flow velocity at station 02074000 Smith River at Eden, N.C. 
DISCHARGE MEASUREMENT DATA FOR STATION 02074000 SMITH RIVER AT EDEN, N.C.

[ft, foot; ft/s, foot per second]

\begin{tabular}{|c|c|c|}
\hline \multicolumn{3}{|c|}{ Discharge measurement date, 06-21-72 } \\
\hline $\begin{array}{l}\text { Distance from } \\
\text { starting point on } \\
\text { left bank } \\
\text { (ft) }\end{array}$ & $\begin{array}{l}\text { Depth } \\
\text { (ft) }\end{array}$ & $\begin{array}{c}\text { Maximum } \\
\text { velocity } \\
\text { (ft/s) }\end{array}$ \\
\hline 28.00 & 0 & 0.00 \\
\hline 50.00 & 7.5 & 2.76 \\
\hline 75.00 & 10 & 2.22 \\
\hline 90.00 & 12 & 0.00 \\
\hline 115.00 & 23 & 3.08 \\
\hline 120.00 & 22.5 & 5.10 \\
\hline 125.00 & 22.5 & 6.69 \\
\hline 130.00 & 23.5 & 6.69 \\
\hline 135.00 & 24 & 7.45 \\
\hline 140.00 & 24 & 7.28 \\
\hline 145.00 & 25.2 & 7.80 \\
\hline 150.00 & 23.5 & 7.28 \\
\hline 155.00 & 24 & 6.98 \\
\hline 160.00 & 23.5 & 3.91 \\
\hline 170.00 & 23.5 & 6.98 \\
\hline 180.00 & 24.5 & 7.28 \\
\hline 185.00 & 25 & 7.13 \\
\hline 190.00 & 23.5 & 5.22 \\
\hline 200.00 & 23.4 & 4.67 \\
\hline 210.00 & 22.2 & 6.31 \\
\hline 220.00 & 22 & 8.00 \\
\hline 230.00 & 23 & 8.19 \\
\hline 240.00 & 22 & 6.69 \\
\hline 250.00 & 22 & 6.83 \\
\hline 260.00 & 23.5 & 6.98 \\
\hline 275.00 & 22.8 & 5.22 \\
\hline 300.00 & 14.3 & 1.27 \\
\hline 320.00 & 0 & 0.00 \\
\hline
\end{tabular}




\section{TAR RIVER AT TARBORO, N.C.}

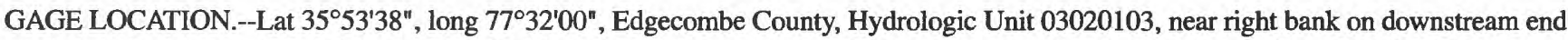
of pier of bridge on U.S. Highway 64 in Tarboro, 6.5 mi downstream of Fishing Creek, and 49.2 mi upstream from Pamlico River at Washington.

MEASUREMENT LOCATION.--Upstream side of bridge on U.S. Highway 64.

DRAINAGE AREA.--2,183 $\mathrm{mi}^{2}$.

PERIOD OF RECORD.--July 1896 to December 1900, October 1931 to current year. Gage-height records at various datums collected at same site since 1905 are contained in reports of National Weather Service, NOAA, U.S. Department of Commerce.

GAGE.--Water-stage recorder with satellite telemetry. Datum of gage is $10.37 \mathrm{ft}$ above sea level. July 1896 to December 1900 , nonrecording gage at Seaboard Coast Line Railroad bridge $600 \mathrm{ft}$ downstream at different datum; Oct. 1 to Dec. 8, 1931, nonrecording gage at site $100 \mathrm{ft}$ upstream at present datum. National Weather Service telephone telemetry at station.

EXTREMES OUTSIDE PERIOD OF RECORD.--Flood of July 27, 1919, reached a stage of $34.0 \mathrm{ft}$, present datum, from flood marks; discharge, $52,800 \mathrm{ft}^{3} / \mathrm{s}$.

\section{DISCHARGE MEASUREMENT SUMMARY}

$\left[\mathrm{ft} / \mathrm{s}\right.$, foot per second; $\mathrm{ft}$, foot; $\mathrm{ft}^{3} / \mathrm{s}$, cubic foot per second]

\begin{tabular}{ccccccc}
\hline $\begin{array}{c}\text { Date } \\
\text { of } \\
\text { measurement }\end{array}$ & $\begin{array}{c}\text { Stream stage } \\
\text { (feet above } \\
\text { datum) }\end{array}$ & $\begin{array}{c}\text { Average } \\
\text { veiocity } \\
(\mathbf{f t} / \mathbf{s})\end{array}$ & $\begin{array}{c}\text { Maximum } \\
\text { velocity } \\
(\mathbf{f t} / \mathbf{s})\end{array}$ & $\begin{array}{c}\text { Maximum } \\
\text { depth } \\
(\mathbf{f t})\end{array}$ & $\begin{array}{c}\text { Discharge } \\
\left(\mathbf{f t}^{\mathbf{3}} / \mathbf{s}\right)\end{array}$ & $\begin{array}{c}\text { Recurrence } \\
\text { interval } \\
(\mathbf{y e a r s})\end{array}$ \\
\hline $04-22-87$ & 28.36 & 2.77 & 4.60 & 31.0 & 25,200 & 10 \\
$09-12-96$ & 22.09 & 2.47 & 4.37 & 24.1 & 14,100 & 2 \\
\hline
\end{tabular}

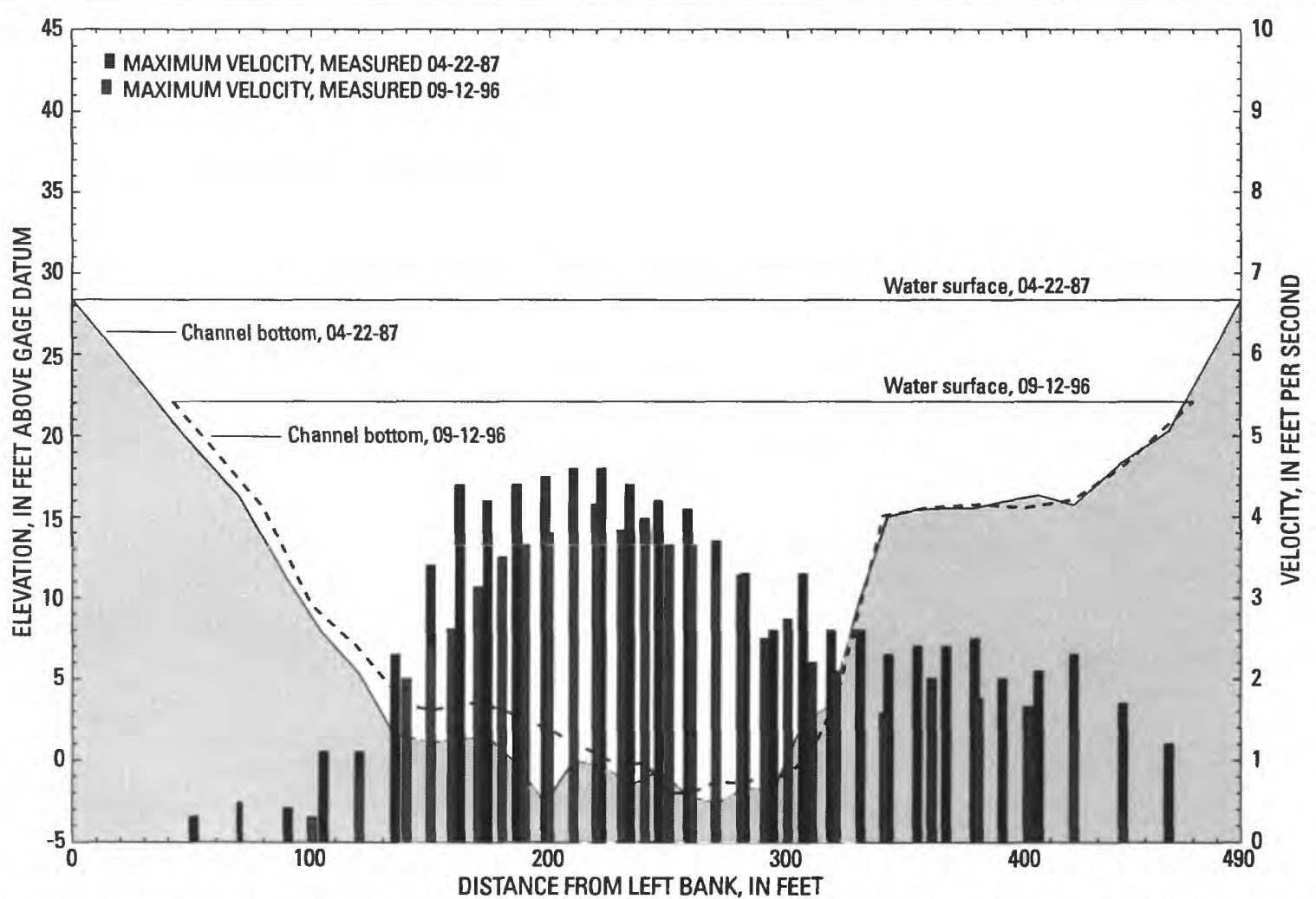

Figure 4. Channel cross sections and maximum flow velocities at station 02083500 Tar River at Tarboro, N.C. 
DISCHARGE MEASUREMENT DATA FOR STATION 02083500 TAR RIVER AT TARBORO, N.C.

[ft, foot; ft/s, foot per second; nd, no data]

\begin{tabular}{|c|c|c|c|c|c|}
\hline \multicolumn{3}{|c|}{ Discharge measurement date, 04-22-87 } & \multicolumn{3}{|c|}{ Discharge measurement date, 09-12-96 } \\
\hline $\begin{array}{l}\text { Distance from } \\
\text { starting point on } \\
\text { left bank } \\
\text { (ft) }\end{array}$ & $\begin{array}{c}\text { Depth } \\
\text { (ft) }\end{array}$ & $\begin{array}{l}\text { Maximum } \\
\text { velocity } \\
\text { (ft/s) }\end{array}$ & $\begin{array}{l}\text { Distance from } \\
\text { starting point on } \\
\text { left bank } \\
\text { (ft) }\end{array}$ & $\begin{array}{c}\text { Depth } \\
\text { (ft) }\end{array}$ & $\begin{array}{c}\text { Maximum } \\
\text { velocity } \\
\text { (ft/s) }\end{array}$ \\
\hline 0.00 & 0 & 0.00 & 42.00 & 0 & 0.00 \\
\hline 50.00 & 8.9 & 0.31 & 80.00 & 6.5 & 0.00 \\
\hline 90.00 & 17.2 & 0.42 & 120.00 & 15.2 & 0.86 \\
\hline 105.00 & 20.6 & 1.10 & 140.00 & 18.7 & 2.00 \\
\hline 120.00 & 23 & 1.10 & 150.00 & 19 & 2.39 \\
\hline 135.00 & 26.8 & 2.30 & 160.00 & 18.8 & 2.61 \\
\hline 186.00 & 28.6 & 4.40 & 200.00 & 20.2 & 3.80 \\
\hline 198.00 & 31 & 4.50 & 210.00 & 21 & 4.37 \\
\hline 210.00 & 28.4 & 4.60 & 220.00 & 21.7 & 4.16 \\
\hline 222.00 & 28.7 & 4.60 & 230.00 & 22.5 & 3.83 \\
\hline 234.00 & 29.9 & 4.40 & 240.00 & 22.2 & 3.98 \\
\hline 246.00 & 29 & 4.20 & 250.00 & 24.1 & 3.65 \\
\hline 258.00 & 30.5 & 4.10 & 260.00 & 24 & 3.65 \\
\hline 270.00 & 31 & 3.70 & 270.00 & 23.4 & 3.28 \\
\hline 282.00 & 30.1 & 3.30 & 280.00 & 23.5 & 3.28 \\
\hline 354.00 & 12.9 & 2.40 & 360.00 & 6.5 & 2.00 \\
\hline 366.00 & 12.8 & 2.40 & 380.00 & 6.3 & 1.76 \\
\hline 378.00 & 12.8 & 2.50 & 400.00 & 6.5 & 1.66 \\
\hline 390.00 & 12.4 & 2.00 & 420.00 & 6 & 1.44 \\
\hline 405.00 & 12 & 2.10 & 440.00 & 4 & 0.39 \\
\hline 420.00 & 12.6 & 2.30 & 470.00 & 0 & 0.00 \\
\hline 440.00 & 10 & 1.70 & nd & nd & nd \\
\hline 460.00 & 8 & 1.20 & nd & nd & nd \\
\hline 490.00 & 0 & 0.00 & nd & nd & nd \\
\hline
\end{tabular}




\section{CONTENTNEA CREEK NEAR LUCAMA, NC}

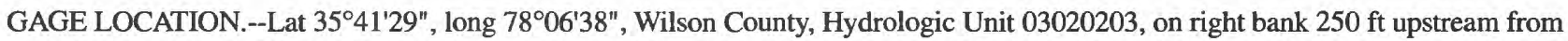
bridge on State Highway 581, 1.0 mi downstream of Buckhorn Reservoir, 1.0 mi upstream from Buckhorn Branch, and 6.5 mi northwest of Lucama.

MEASUREMENT LOCATION.--Upstream side of bridge on State Highway 581.

DRAINAGE AREA.--161 $\mathrm{mi}^{2}$.

PERIOD OF RECORD.--September 1964 to current year.

GAGE.--Water-stage recorder. Datum of gage is $117.43 \mathrm{ft}$ above sea level (levels by U.S. Army Corps of Engineers). Satellite telemetry at station.

\section{DISCHARGE MEASUREMENT SUMMARY}

[ft/s, foot per second; $\mathrm{ft}$, foot; $\mathrm{ft}^{3} / \mathrm{s}$, cubic foot per second]

\begin{tabular}{ccccccc}
\hline $\begin{array}{c}\text { Date } \\
\text { of } \\
\text { measurement }\end{array}$ & $\begin{array}{c}\text { Stream stage } \\
\text { (feet above } \\
\text { datum) }\end{array}$ & $\begin{array}{c}\text { Average } \\
\text { velocity } \\
(\mathrm{ft} / \mathrm{s})\end{array}$ & $\begin{array}{c}\text { Maximum } \\
\text { velocity } \\
(\mathrm{ft} / \mathrm{s})\end{array}$ & $\begin{array}{c}\text { Maximum } \\
\text { depth } \\
\mathbf{( f t )}\end{array}$ & $\begin{array}{c}\text { Discharge } \\
\left(\mathrm{ft}^{3} / \mathbf{s}\right)\end{array}$ & $\begin{array}{c}\text { Recurrence } \\
\text { interval } \\
(\mathrm{years})\end{array}$ \\
\hline $10-06-64$ & 16.09 & 3.01 & 5.92 & 15.7 & 5,670 & 100 \\
$02-05-98$ & 12.27 & 2.33 & 4.20 & 11.7 & 2,380 & $2-5$ \\
\hline
\end{tabular}

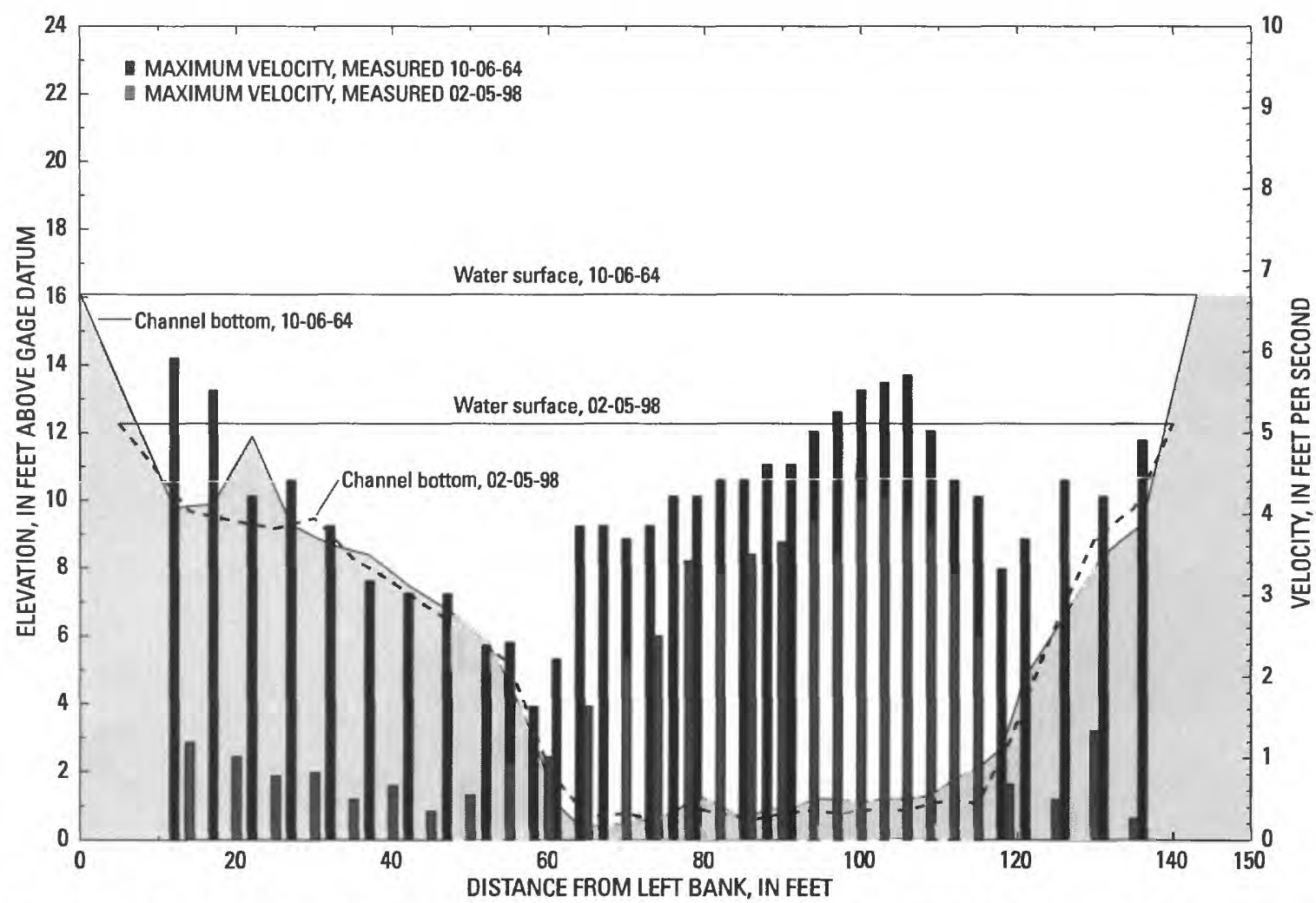

Figure 5. Channel cross sections and maximum flow velocities at station 02090380 Contentnea Creek near Lucama, N.C. 
DISCHARGE MEASUREMENT DATA FOR STATION 02090380 CONTENTNEA CREEK NEAR LUCAMA, N.C.

[ft, foot; ft/s, foot per second; nd, no data]

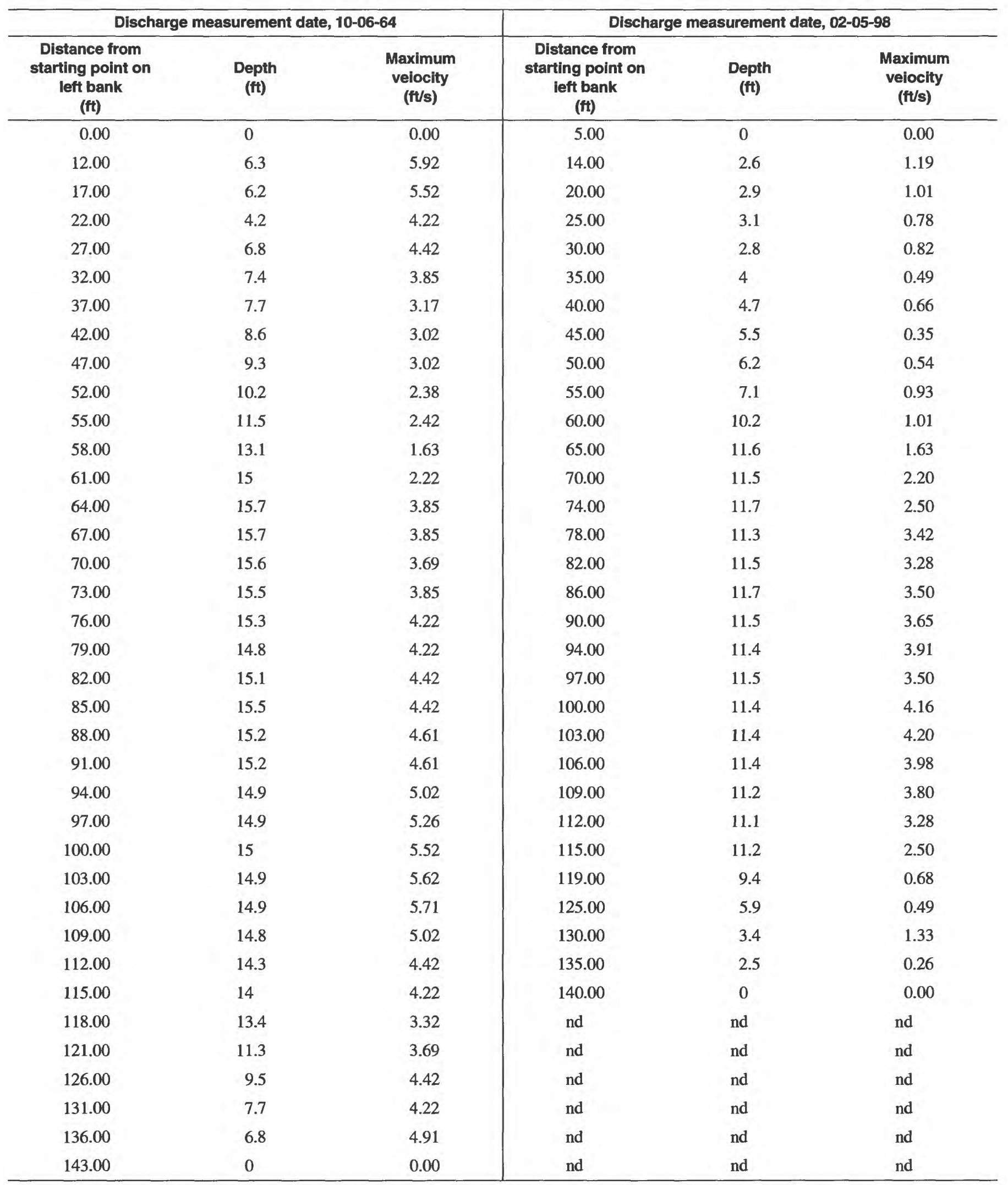




\section{NEW RIVER NEAR GUM BRANCH, N.C.}

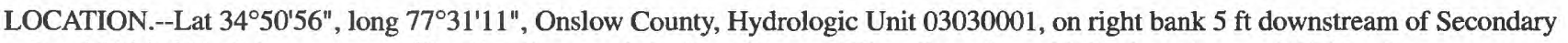
Road 1314, 0.7 mi downstream of Jenkins Swamp, 1.8 mi southwest of Gum Branch, and 3.8 mi southeast of Richlands.

MEASUREMENT LOCATION.--Downstream side of bridge on Secondary Road 1314.

DRAINAGE AREA.--94 $\mathrm{mi}^{2}$.

PERIOD OF RECORD.--August 1949 to September 1973. July 1987 to current year.

GAGE.--Water-stage recorder. Datum of gage is sea level (levels by U.S. Army Corps of Engineers). Aug. 19, 1949, to Mar. 22, 1950, nonrecording gage and Mar. 23, 1950, to Mar. 25, 1969, water-stage recorder at site 0.2 mi upstream and datum of $2.52 \mathrm{ft}$ above mean sea level. Mar. 26, 1969, to Sept. 1973 water-stage recorder at present site and datum. Satellite telemetry at station.

EXTREMES OUTSIDE PERIOD OF RECORD.--Flood of 1908 reached a stage of about $18 \mathrm{ft}$ at former site and datum, from information by local resident.

\section{DISCHARGE MEASUREMENT SUMMARY}

[ft/s, foot per second; ft, foot: $\mathrm{ft}^{3} / \mathrm{s}$, cubic foot per second]

\begin{tabular}{ccccccc}
\hline $\begin{array}{c}\text { Date } \\
\text { of } \\
\text { measurement }\end{array}$ & $\begin{array}{c}\text { Stream stage } \\
\text { (feet above } \\
\text { datum) }\end{array}$ & $\begin{array}{c}\text { Average } \\
\text { velocity } \\
(\mathrm{ft} / \mathrm{s})\end{array}$ & $\begin{array}{c}\text { Maximum } \\
\text { velocity } \\
(\mathrm{ft} / \mathrm{s})\end{array}$ & $\begin{array}{c}\text { Maximum } \\
\text { depth } \\
(\mathrm{ft})\end{array}$ & $\begin{array}{c}\text { Discharge } \\
\left(\mathrm{ft}^{3} / \mathbf{s}\right)\end{array}$ & $\begin{array}{c}\text { Recurrence } \\
\text { interval } \\
(\text { (years) }\end{array}$ \\
\hline $09-07-96$ & 19.42 & 2.06 & 4.07 & 20.8 & 2,680 & 5 \\
$08-28-98$ & 18.21 & 2.00 & 3.82 & 19.5 & 2,320 & $2-5$ \\
\hline
\end{tabular}

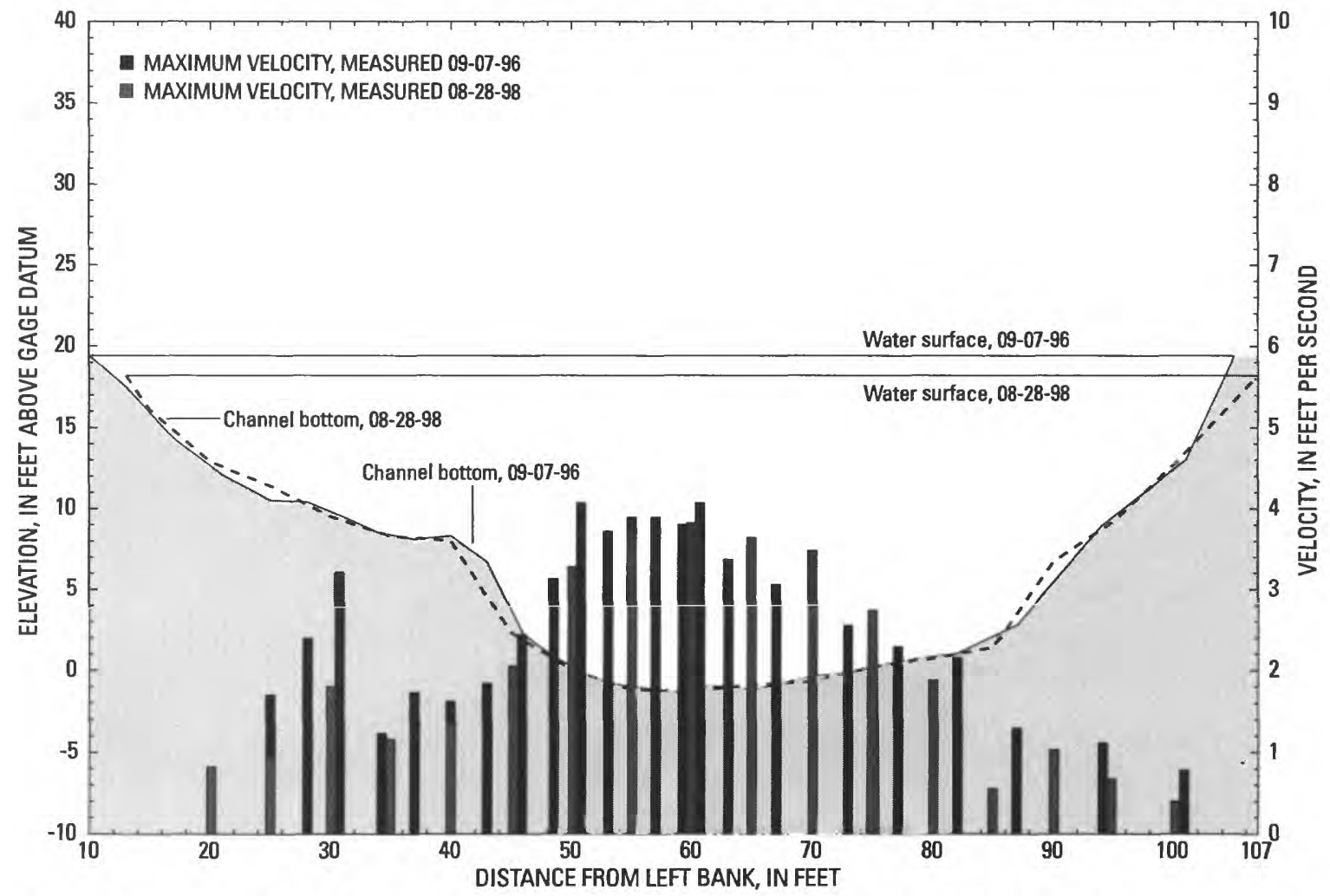

Figure 6. Channel cross sections and maximum flow velocities at station 02093000 New River near Gum Branch, N.C. 
DISCHARGE MEASUREMENT DATA FOR STATION 02093000 NEW RIVER NEAR GUM BRANCH, N.C.

[ft, foot; ft/s, foot per second; nd, no data]

\begin{tabular}{|c|c|c|c|c|c|}
\hline \multicolumn{3}{|c|}{ Discharge measurement date, 09-07-96 } & \multicolumn{3}{|c|}{ Discharge measurement date, $08-28-98$} \\
\hline $\begin{array}{l}\text { Distance from } \\
\text { starting point on } \\
\text { left bank } \\
\text { (ft) }\end{array}$ & $\begin{array}{l}\text { Depth } \\
\text { (ft) }\end{array}$ & $\begin{array}{l}\text { Maximum } \\
\text { velocity } \\
\text { (tt/s) }\end{array}$ & $\begin{array}{l}\text { Distance from } \\
\text { starting point on } \\
\text { left bank } \\
\text { (ft) }\end{array}$ & $\begin{array}{c}\text { Depth } \\
\text { (ft) }\end{array}$ & $\begin{array}{l}\text { Maximum } \\
\text { velocity } \\
\text { (ft/s) }\end{array}$ \\
\hline 10.00 & 0 & 0.00 & 13.00 & 0 & 0.00 \\
\hline 13.00 & 1.9 & -1.26 & 15.00 & 2.1 & -0.47 \\
\hline 17.00 & 5.1 & -0.65 & 20.00 & 5.3 & 0.82 \\
\hline 21.00 & 7.3 & -0.70 & 25.00 & 6.8 & 0.93 \\
\hline 25.00 & 8.9 & 1.70 & 30.00 & 8.7 & 1.80 \\
\hline 28.00 & 9 & 2.39 & 35.00 & 9.9 & 1.16 \\
\hline 31.00 & 9.9 & 3.21 & 40.00 & 10.2 & 1.35 \\
\hline 34.00 & 10.9 & 1.23 & 45.00 & 15.9 & 2.05 \\
\hline 37.00 & 11.3 & 1.73 & 50.00 & 18.2 & 3.28 \\
\hline 40.00 & 11.1 & 1.62 & 55.00 & 19.4 & 3.72 \\
\hline 43.00 & 12.7 & 1.84 & 60.00 & 19.5 & 3.82 \\
\hline 46.00 & 17.2 & 2.44 & 65.00 & 19.1 & 3.64 \\
\hline 48.50 & 18.6 & 3.13 & 70.00 & 18.9 & 3.48 \\
\hline 51.00 & 19.6 & 4.07 & 75.00 & 18 & 2.74 \\
\hline 53.00 & 20.2 & 3.72 & 80.00 & 17.5 & 1.88 \\
\hline 55.00 & 20.4 & 3.89 & 85.00 & 16.8 & 0.55 \\
\hline 57.00 & 20.6 & 3.89 & 90.00 & 11.5 & 1.03 \\
\hline 59.00 & 20.8 & 3.80 & 95.00 & 9 & 0.67 \\
\hline 61.00 & 20.4 & 4.07 & 100.00 & 5.5 & 0.40 \\
\hline 63.00 & 20.4 & 3.37 & 107.00 & 0 & 0.00 \\
\hline 65.00 & 20.6 & 3.50 & nd & nd & nd \\
\hline 67.00 & 20.3 & 3.06 & nd & nd & nd \\
\hline 70.00 & 19.8 & 2.80 & nd & nd & nd \\
\hline 73.00 & 19.6 & 2.55 & nd & nd & nd \\
\hline 77.00 & 18.9 & 2.29 & nd & nd & nd \\
\hline 82.00 & 18.4 & 2.15 & nd & nd & nd \\
\hline 87.00 & 16.6 & 1.92 & nd & nd & nd \\
\hline 94.00 & 10.5 & 1.11 & nd & nd & nd \\
\hline 101.00 & 6.4 & 0.78 & nd & nd & nd \\
\hline 105.00 & 0 & 0.00 & nd & nd & nd \\
\hline
\end{tabular}


GAGE LOCATION.--Lat 3605'13", long 79²2'02", Alamance County, Hydrologic unit 03030002 , on left bank at Haw River, $650 \mathrm{ft}$ downstream of Southern Railway bridge, $800 \mathrm{ft}$ downstream of bridge on U.S. Highway 70 and State Highway 49, and 3 mi downstream of Stony Creek.

MEASUREMENT LOCATION.--Upstream side of bridge on U.S. Highway 70 and State Highway 49.

DRAINAGE AREA.--606 $\mathrm{mi}^{2}$.

PERIOD OF RECORD.--October 1928 to current year.

GAGE.--Water-stage recorder. Datum of gage is $471.69 \mathrm{ft}$ above sea level. U.S. Army Corps of Engineers satellite telemetry at station.

\section{DISCHARGE MEASUREMENT SUMMARY}

[ft/s, foot per second; $\mathrm{ft}$, foot; $\mathrm{ft}^{3} / \mathrm{s}$, cubic foot per second]

\begin{tabular}{ccccccc}
\hline $\begin{array}{c}\text { Date } \\
\text { of } \\
\text { measurement }\end{array}$ & $\begin{array}{c}\text { Stream stage } \\
\text { (feet above } \\
\text { datum) }\end{array}$ & $\begin{array}{c}\text { Average } \\
\text { velocity } \\
\text { (ft/s) }\end{array}$ & $\begin{array}{c}\text { Maximum } \\
\text { velocity } \\
(\mathrm{ft} / \mathbf{s})\end{array}$ & $\begin{array}{c}\text { Maximum } \\
\text { depth } \\
(\mathbf{f t})\end{array}$ & $\begin{array}{c}\text { Discharge } \\
\left(\mathrm{ft}^{\mathbf{3}} / \mathbf{s}\right)\end{array}$ & $\begin{array}{c}\text { Recurrence } \\
\text { interval } \\
(\text { years) }\end{array}$ \\
\hline $09-07-96$ & 25.76 & 4.45 & 8.46 & 26.0 & 22,600 & 10 \\
$02-05-98$ & 15.89 & 2.98 & 5.48 & 15.8 & 7,800 & $1-2$ \\
\hline
\end{tabular}

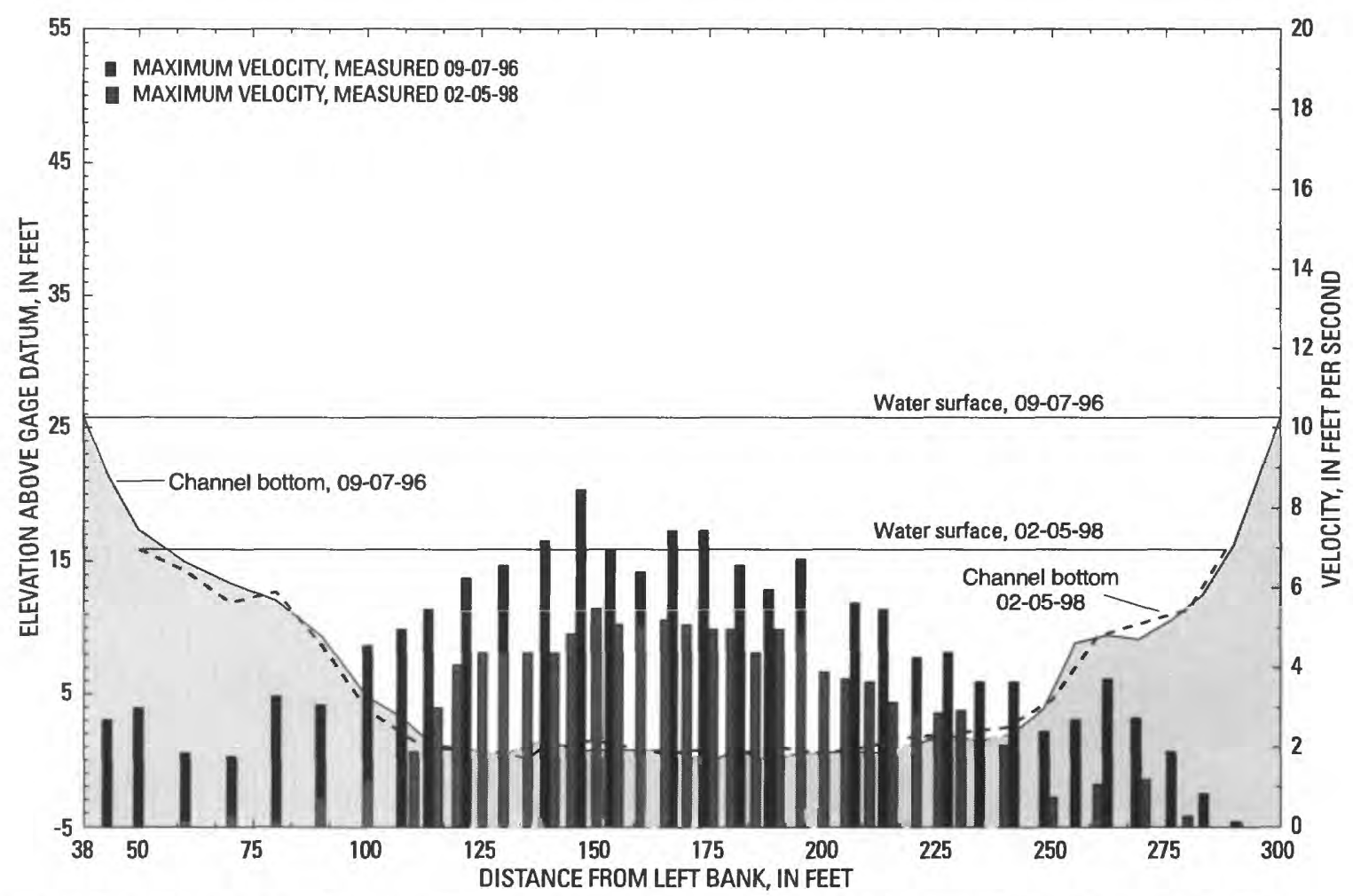

Figure 7. Channel cross sections and flow velocities at station 02096500 Haw River at Haw River, N.C. 
DISCHARGE MEASUREMENT DATA FOR STATION 02096500 HAW RIVER AT HAW RIVER, N.C.

[ft, foot; ft/s, foot per second; nd, no data]

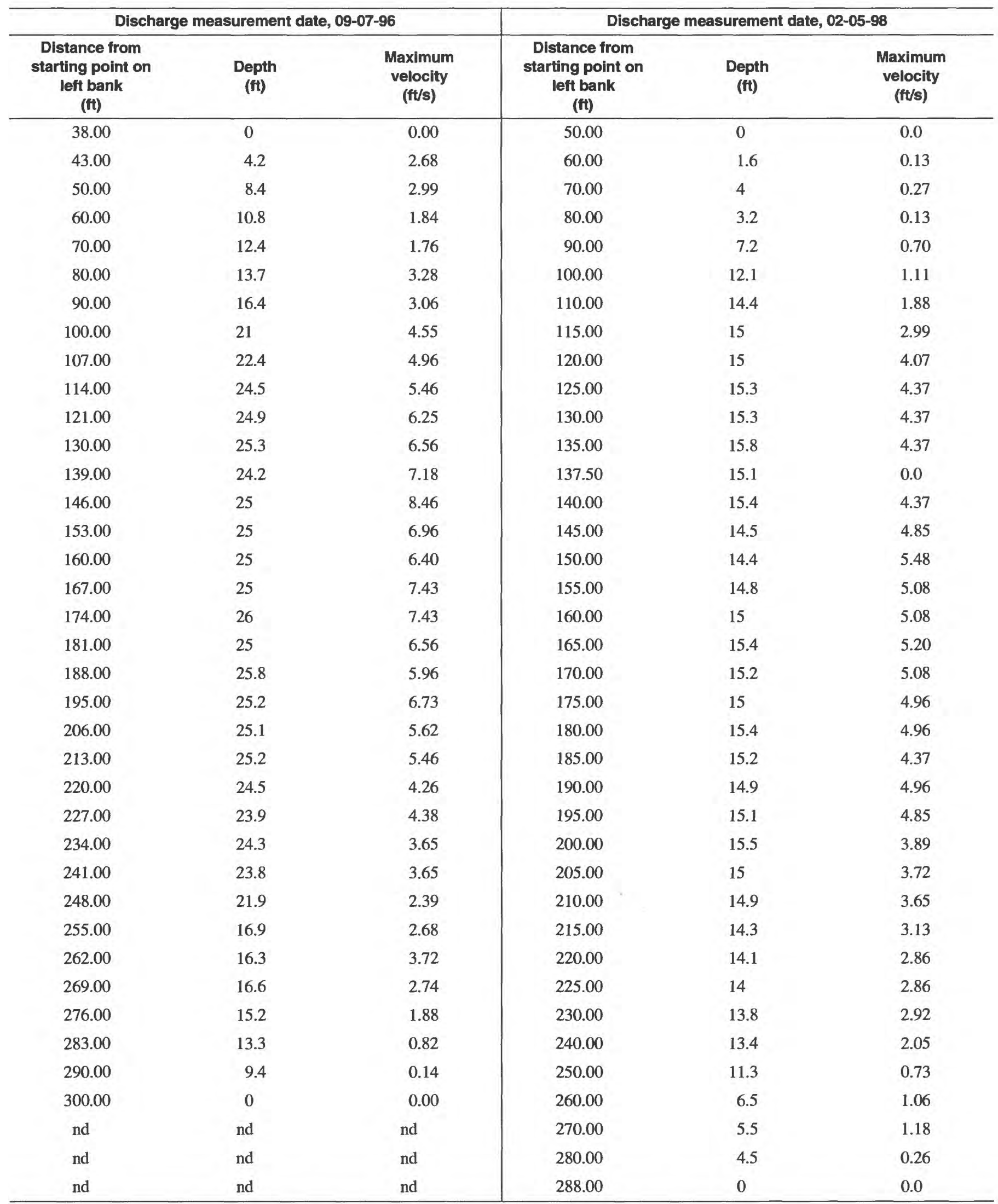




\section{ROCKFISH CREEK AT RAEFORD, NC}

GAGE LOCATION.--Lat 34 $59^{\prime} 55^{\prime \prime}$, long 79 12'55", Hoke County, Hydrologic Unit 03030004, at upstream side of bridge on U.S. Highway $401,1.0 \mathrm{mi}$ downstream of Nicholsons Creek, and 1.0 mile north of Raeford.

MEASUREMENT LOCATION.--Upstream side of bridge on U.S. Highway 401.

DRAINAGE AREA.--93.1 $\mathrm{mi}^{2}$.

PERIOD OF RECORD.--July 1988 to current year.

GAGE.--Water-stage recorder. Elevation of gage is $178 \mathrm{ft}$ above sea level, from topographic map. Satellite telemetry at station.

\section{DISCHARGE MEASUREMENT SUMMARY}

[ft/s, foot per second; $\mathrm{ft}$, foot; $\mathrm{ft}^{3} / \mathrm{s}$, cubic foot per second]

\begin{tabular}{|c|c|c|c|c|c|c|}
\hline $\begin{array}{c}\text { Date } \\
\text { of } \\
\text { measurement }\end{array}$ & $\begin{array}{c}\text { Stream stage } \\
\text { (feet above } \\
\text { datum) }\end{array}$ & $\begin{array}{c}\text { Average } \\
\text { velocity } \\
\text { (ft/s) }\end{array}$ & $\begin{array}{c}\text { Maximum } \\
\text { velocity } \\
(\mathrm{ft} / \mathrm{s})\end{array}$ & $\begin{array}{l}\text { Maximum } \\
\text { depth } \\
\text { (ft) }\end{array}$ & $\begin{array}{c}\text { Discharge } \\
\left(\mathrm{ft}^{3} / \mathrm{s}\right)\end{array}$ & $\begin{array}{c}\text { Recurrence } \\
\text { interval } \\
\text { (years) }\end{array}$ \\
\hline $09-07-96$ & 8.26 & 2.24 & 4.37 & 8.4 & 994 & $\begin{array}{l}\text { Insufficient data } \\
\text { to determine }\end{array}$ \\
\hline $07-25-97$ & 8.14 & 2.40 & 3.72 & 8.1 & 971 & $\begin{array}{l}\text { Insufficient data } \\
\text { to determine }\end{array}$ \\
\hline
\end{tabular}

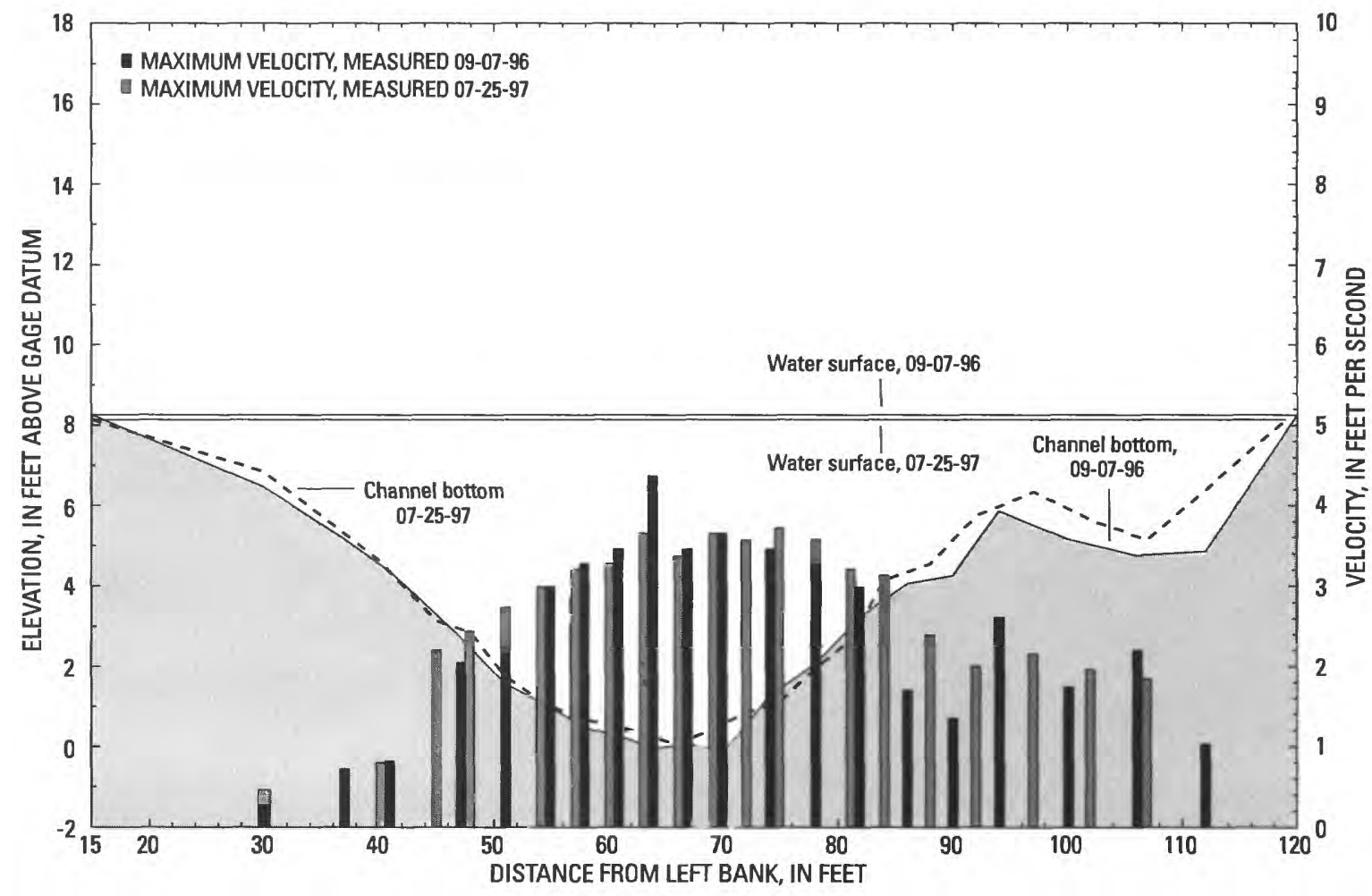

Figure 8. Channel cross sections and maximum flow velocities at station 02104220 Rockfish Creek at Raeford, N.C. 
DISCHARGE MEASUREMENT DATA FOR STATION 02104220 ROCKFISH CREEK AT RAEFORD, N.C.

[ft, foot; ft/s, foot per second; nd, no data]

\begin{tabular}{|c|c|c|c|c|c|}
\hline \multicolumn{3}{|c|}{ Discharge measurement date, 09-07-96 } & \multicolumn{3}{|c|}{ Discharge measurement date, 07-25-97 } \\
\hline $\begin{array}{l}\text { Distance from } \\
\text { starting point on } \\
\text { left bank } \\
\text { (ft) }\end{array}$ & $\begin{array}{c}\text { Depth } \\
\text { (ft) }\end{array}$ & $\begin{array}{l}\text { Maximum } \\
\text { velocity } \\
(\mathrm{ft} / \mathrm{s})\end{array}$ & $\begin{array}{l}\text { Distance from } \\
\text { starting point on } \\
\text { left bank } \\
\text { (ft) }\end{array}$ & $\begin{array}{c}\text { Depth } \\
\text { (ft) }\end{array}$ & $\begin{array}{l}\text { Maximum } \\
\text { veiocity } \\
\text { (ft/s) }\end{array}$ \\
\hline 15.00 & 0 & 0.00 & 15.00 & 0 & 0.00 \\
\hline 30.00 & 1.8 & 0.27 & 30.00 & 1.3 & 0.46 \\
\hline 41.00 & 3.9 & 0.82 & 45.00 & 5 & 2.20 \\
\hline 47.00 & 5.5 & 2.05 & 48.00 & 5.3 & 2.44 \\
\hline 51.00 & 6.7 & 2.20 & 51.00 & 6.4 & 2.74 \\
\hline 55.00 & 7.3 & 2.99 & 54.00 & 7 & 2.99 \\
\hline 67.00 & 8.2 & 3.46 & 66.00 & 8.1 & 3.37 \\
\hline 70.00 & 8.4 & 3.65 & 69.00 & 7.7 & 3.65 \\
\hline 74.00 & 7 & 3.46 & 72.00 & 7.3 & 3.57 \\
\hline 78.00 & 6.2 & 3.28 & 75.00 & 7 & 3.72 \\
\hline 82.00 & 5 & 2.99 & 78.00 & 6.2 & 3.57 \\
\hline 86.00 & 4.2 & 1.70 & 81.00 & 5.5 & 3.21 \\
\hline 90.00 & 4 & 1.35 & 84.00 & 4 & 3.13 \\
\hline 94.00 & 2.4 & 2.61 & 88.00 & 3.6 & 2.39 \\
\hline 100.00 & 3.1 & 1.74 & 92.00 & 2.4 & 2.00 \\
\hline
\end{tabular}




\section{YADKIN RIVER AT ELKIN, NC}

GAGE LOCATION.--Lat 36¹4'30", long 8050'49", Yadkin County, Hydrologic Unit 03040101, on right bank at downstream side of bridge on U.S. Highway 21 at Elkin, 0.3 mi downstream of Elkin River, and 362 mi upstream from mouth of Pee Dee River in Winyah Bay.

MEASUREMENT LOCATION.--Upstream side of Main Street bridge, $500 \mathrm{ft}$ upstream of bridge on U.S. Highway 21.

DRAINAGE AREA.--869 $\mathrm{mi}^{2}$.

PERIOD OF RECORD.--April 1964 to current year.

GAGE.--Water-stage recorder. Datum of gage is $866.03 \mathrm{ft}$ above sea level. Prior to Aug. 28, 1964, nonrecording gage on upstream side of bridge at same datum. U.S. Army Corps of Engineers satellite telemetry at station.

EXTREMES OUTSIDE PERIOD OF RECORD.--Flood of July 1916 reached a stage of $36.0 \mathrm{ft}$, from information by North Carolina State Highway Commission. Flood of August 1940 reached a stage of $37.5 \mathrm{ft}$.

\section{DISCHARGE MEASUREMENT SUMMARY}

[ft/s, foot per second; ft, foot; $\mathrm{ft}^{3} / \mathrm{s}$, cubic foot per second]

\begin{tabular}{ccccccc}
\hline $\begin{array}{c}\text { Date } \\
\text { of } \\
\text { measurement }\end{array}$ & $\begin{array}{c}\text { Stream stage } \\
\text { (feet above } \\
\text { datum) }\end{array}$ & $\begin{array}{c}\text { Average } \\
\text { velocity } \\
(\mathrm{ft} / \mathrm{s})\end{array}$ & $\begin{array}{c}\text { Maximum } \\
\text { velocity } \\
(\mathrm{ft} / \mathrm{s})\end{array}$ & $\begin{array}{c}\text { Maximum } \\
\text { depth } \\
(\mathrm{ft})\end{array}$ & $\begin{array}{c}\text { Discharge } \\
\left(\mathrm{ft}^{3} / \mathrm{s}\right)\end{array}$ & $\begin{array}{c}\text { Recurrence } \\
\text { interval } \\
(\text { years })\end{array}$ \\
\hline $08-17-94$ & 22.52 & 5.36 & 7.97 & 28.5 & 26,600 & $10-25$ \\
\hline
\end{tabular}

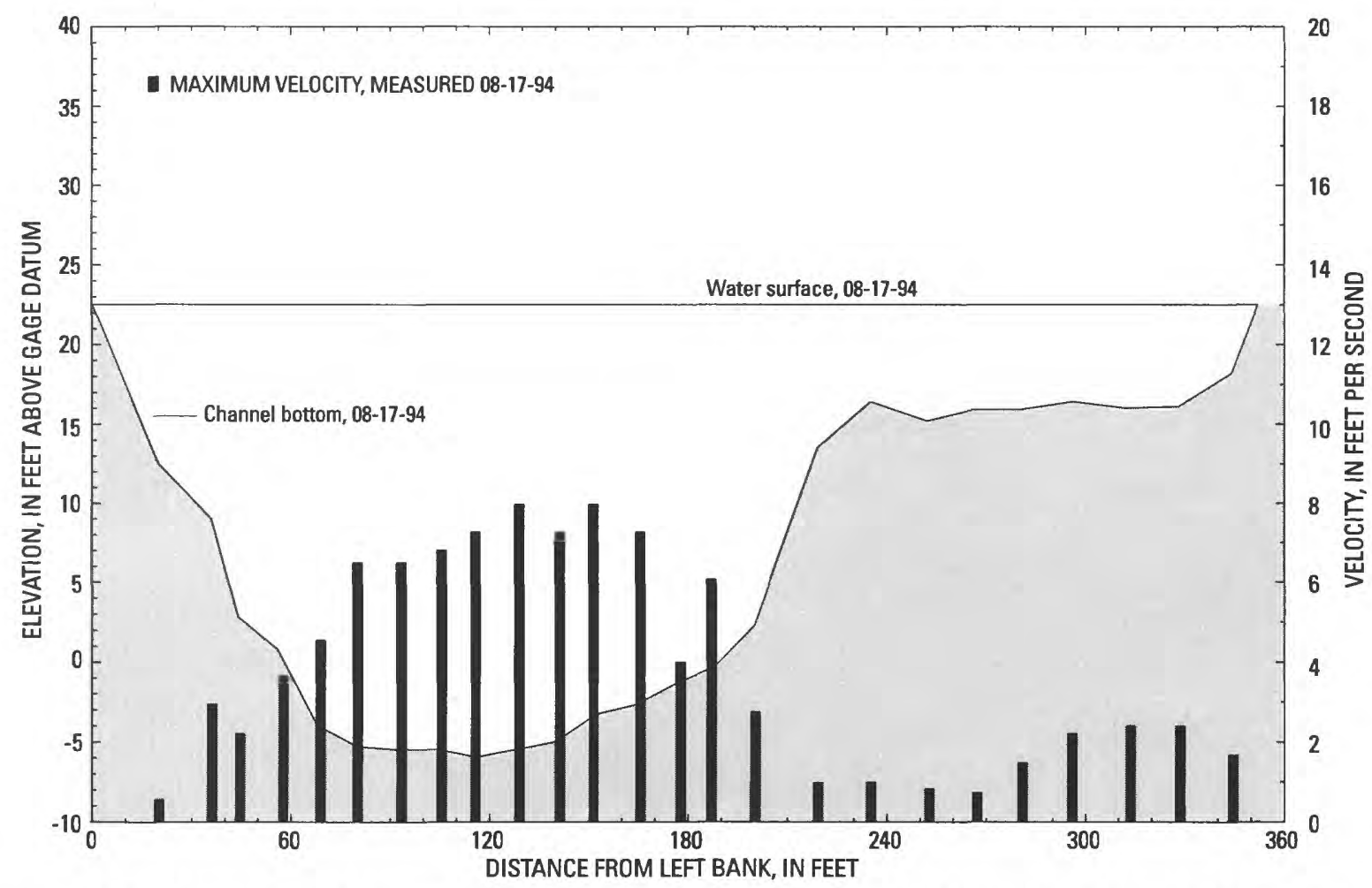

Figure 9. Channel cross section and maximum flow velocity at station 02112250 Yadkin River at Elkin, N.C. 
DISCHARGE MEASUREMENT DATA FOR STATION 02112250 YADKIN RIVER AT ELKIN, N.C.

[ft, foot; ft/s, foot per second]

\begin{tabular}{|c|c|c|}
\hline \multicolumn{3}{|c|}{ Discharge measurement date, 08-17-94 } \\
\hline $\begin{array}{l}\text { Distance from } \\
\text { starting point on } \\
\text { left bank } \\
\text { (ft) }\end{array}$ & $\begin{array}{l}\text { Depth } \\
\text { (ft) }\end{array}$ & $\begin{array}{c}\text { Maximum } \\
\text { velocity } \\
(\mathrm{ft} / \mathrm{s})\end{array}$ \\
\hline 0.00 & 0 & 0.00 \\
\hline 20.00 & 10 & 0.55 \\
\hline 36.00 & 13.5 & 2.94 \\
\hline 44.00 & 19.7 & 2.20 \\
\hline 56.00 & 21.7 & 3.65 \\
\hline 68.00 & 26.5 & 4.55 \\
\hline 80.00 & 27.8 & 6.49 \\
\hline 92.00 & 28 & 6.49 \\
\hline 104.00 & 28 & 6.81 \\
\hline 116.00 & 28.5 & 7.26 \\
\hline 128.00 & 28 & 7.97 \\
\hline 140.00 & 27.5 & 7.26 \\
\hline 152.00 & 25.8 & 7.97 \\
\hline 164.00 & 25.2 & 7.26 \\
\hline 176.00 & 23.9 & 4.00 \\
\hline 188.00 & 22.8 & 6.06 \\
\hline 200.00 & 20.2 & 2.74 \\
\hline 219.00 & 9 & 0.97 \\
\hline 235.00 & 6.1 & 0.99 \\
\hline 252.00 & 7.3 & 0.82 \\
\hline 266.00 & 6.6 & 0.72 \\
\hline 280.00 & 6.6 & 1.47 \\
\hline 296.00 & 6.1 & 2.20 \\
\hline 312.00 & 6.5 & 2.39 \\
\hline 328.00 & 6.4 & 2.39 \\
\hline 344.00 & 4.3 & 1.66 \\
\hline 352.00 & 0 & 0.00 \\
\hline
\end{tabular}




\section{FISHER RIVER NEAR COPELAND, NC}

GAGE LOCATION.--Lat $36^{\circ} 21^{\prime 2} 26^{\prime \prime}$, long $80^{\circ} 41^{\prime} 10^{\prime \prime}$, Surry County, Hydrologic Unit 03040101 , on left bank $500 \mathrm{ft}$ upstream from bridge on State Highway 268, 1 mi upstream from Cody Creek, and 2 mi northwest of Copeland.

MEASUREMENT LOCATION.--Upstream side of bridge on State Highway 268, $500 \mathrm{ft}$ downstream of gage.

DRAINAGE AREA.--128 $\mathrm{mi}^{2}$.

PERIOD OF RECORD.--October 1931 to current year.

GAGE.--Water-stage recorder. Datum of gage is $913 \mathrm{ft}$ above sea level, by barometer. Prior to Sept. 5, 1936, twice daily readings at same site and datum. Satellite telemetry at station.

\section{DISCHARGE MEASUREMENT SUMMARY}

[ft/s, foot per second; $\mathrm{ft}$, foot; $\mathrm{ft}^{3} / \mathrm{s}$, cubic foot per second]

\begin{tabular}{ccccccc}
\hline $\begin{array}{c}\text { Date } \\
\text { of } \\
\text { measurement }\end{array}$ & $\begin{array}{c}\text { Stream stage } \\
\text { (feet above } \\
\text { datum) }\end{array}$ & $\begin{array}{c}\text { Average } \\
\text { velocity } \\
(\mathrm{ft} / \mathrm{s})\end{array}$ & $\begin{array}{c}\text { Maximum } \\
\text { velocity } \\
(\mathrm{ft} / \mathrm{s})\end{array}$ & $\begin{array}{c}\text { Maximum } \\
\text { depth } \\
\text { (ft) }\end{array}$ & $\begin{array}{c}\text { Discharge } \\
\left(\mathrm{ft}^{3} / \mathrm{s}\right)\end{array}$ & $\begin{array}{c}\text { Recurrence } \\
\text { interval } \\
(\mathrm{years})\end{array}$ \\
\hline $03-02-94$ & 8.24 & 5.71 & 9.10 & 9.0 & 4,250 & $1-2$ \\
\hline
\end{tabular}

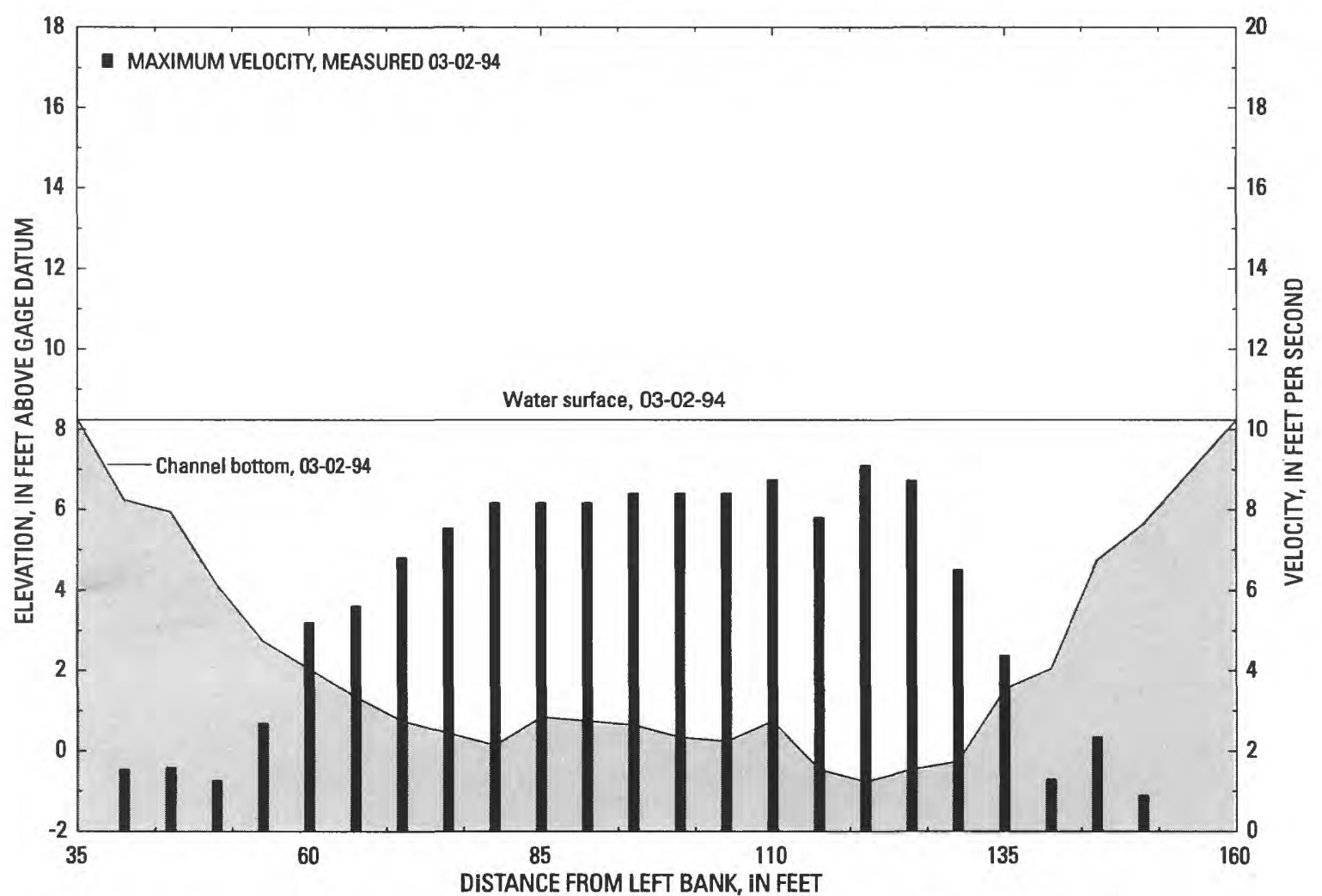

Figure 10. Channel cross section and maximum flow velocity at station 02113000 Fisher River near Copeland, N.C. 
DISCHARGE MEASUREMENT DATA FOR STATION 02113000 FISHER RIVER NEAR COPELAND, N.C.

[ft, foot; ft/s, foot per second]

\begin{tabular}{|c|c|c|}
\hline \multicolumn{3}{|c|}{ Discharge measurement date, 03-02-94 } \\
\hline $\begin{array}{l}\text { Distance from } \\
\text { starting point on } \\
\text { left bank } \\
\text { (ft) }\end{array}$ & $\begin{array}{l}\text { Depth } \\
\text { (ft) }\end{array}$ & $\begin{array}{l}\text { Maximum } \\
\text { veiocity } \\
\text { (ft/s) }\end{array}$ \\
\hline 35.00 & 0 & -99.99 \\
\hline 40.00 & 2 & 1.54 \\
\hline 45.00 & 2.3 & 1.58 \\
\hline 50.00 & 4.1 & 1.26 \\
\hline 55.00 & 5.5 & 2.68 \\
\hline 60.00 & 6.2 & 5.20 \\
\hline 65.00 & 6.9 & 5.61 \\
\hline 70.00 & 7.5 & 6.81 \\
\hline 75.00 & 7.8 & 7.54 \\
\hline 80.00 & 8.1 & 8.17 \\
\hline 85.00 & 7.4 & 8.17 \\
\hline 90.00 & 7.5 & 8.17 \\
\hline 95.00 & 7.6 & 8.40 \\
\hline 100.00 & 7.9 & 8.40 \\
\hline 105.00 & 8 & 8.40 \\
\hline 110.00 & 7.5 & 8.74 \\
\hline 115.00 & 8.7 & 7.80 \\
\hline 120.00 & 9 & 9.10 \\
\hline 125.00 & 8.7 & 8.72 \\
\hline 130.00 & 8.5 & 6.50 \\
\hline 135.00 & 6.7 & 4.37 \\
\hline 140.00 & 6.2 & 1.29 \\
\hline 145.00 & 3.5 & 2.34 \\
\hline 150.00 & 2.6 & 0.89 \\
\hline 160.00 & 0 & 0.0 \\
\hline
\end{tabular}




\section{PEE DEE RIVER NEAR ROCKINGHAM, N.C.}

GAGE LOCATION.--Lat 3456'46", long 7952'11", Richmond County, Hydrologic Unit 03040201, on left bank at bridge on U.S. Highway 74, 2.5 mi upstream from Falling Creek, 3.3 mi downstream of Blewett Falls hydroelectric plant, 6 mi west of Rockingham, and 192 mi upstream from mouth in Winyah Bay.

MEASUREMENT LOCATION.--Upstream side of bridge on west-bound lanes of U.S. Highway 74.

DRAINAGE AREA.--6,863 $\mathrm{mi}^{2}$.

PERIOD OF RECORD.--August 1906 to January 1912, October 1927 to current year. August 1906 to January 1912 published as "Yadkin River near Pee Dee, NC."

GAGE.--Water-stage recorder with satellite telemetry. Datum of gage is $120.68 \mathrm{ft}$ above sea level (levels by U.S. Army Corps of Engineers).August 1906 to January 1912 nonrecording gage at site 3.3 mi upstream at different datum. Sept. 1927 to Sept. 30, 1931 , waterstage recorder at present site at $121.68 \mathrm{ft}$. Telephone telemetry at station.

\section{DISCHARGE MEASUREMENT SUMMARY}

[ft/s, foot per second; $\mathrm{ft}$, foot; $\mathrm{ft}^{3} / \mathrm{s}$, cubic foot per second]

\begin{tabular}{ccccccc}
\hline $\begin{array}{c}\text { Date } \\
\text { of } \\
\text { measurement }\end{array}$ & $\begin{array}{c}\text { Stream stage } \\
\text { (feet above } \\
\text { datum) }\end{array}$ & $\begin{array}{c}\text { Average } \\
\text { velocity } \\
(\mathbf{f t} / \mathbf{s})\end{array}$ & $\begin{array}{c}\text { Maximum } \\
\text { velocity } \\
(\mathrm{ft} / \mathrm{s})\end{array}$ & $\begin{array}{c}\text { Maximum } \\
\text { depth } \\
(\mathrm{ft})\end{array}$ & $\begin{array}{c}\text { Discharge } \\
\left(\mathrm{ft}^{3} / \mathbf{s}\right)\end{array}$ & $\begin{array}{c}\text { Recurrence } \\
\text { interval } \\
(\text { years })\end{array}$ \\
\hline $07-24-97$ & 14.94 & 6.88 & 9.89 & 27.2 & 94,900 & $2-5$ \\
\hline
\end{tabular}

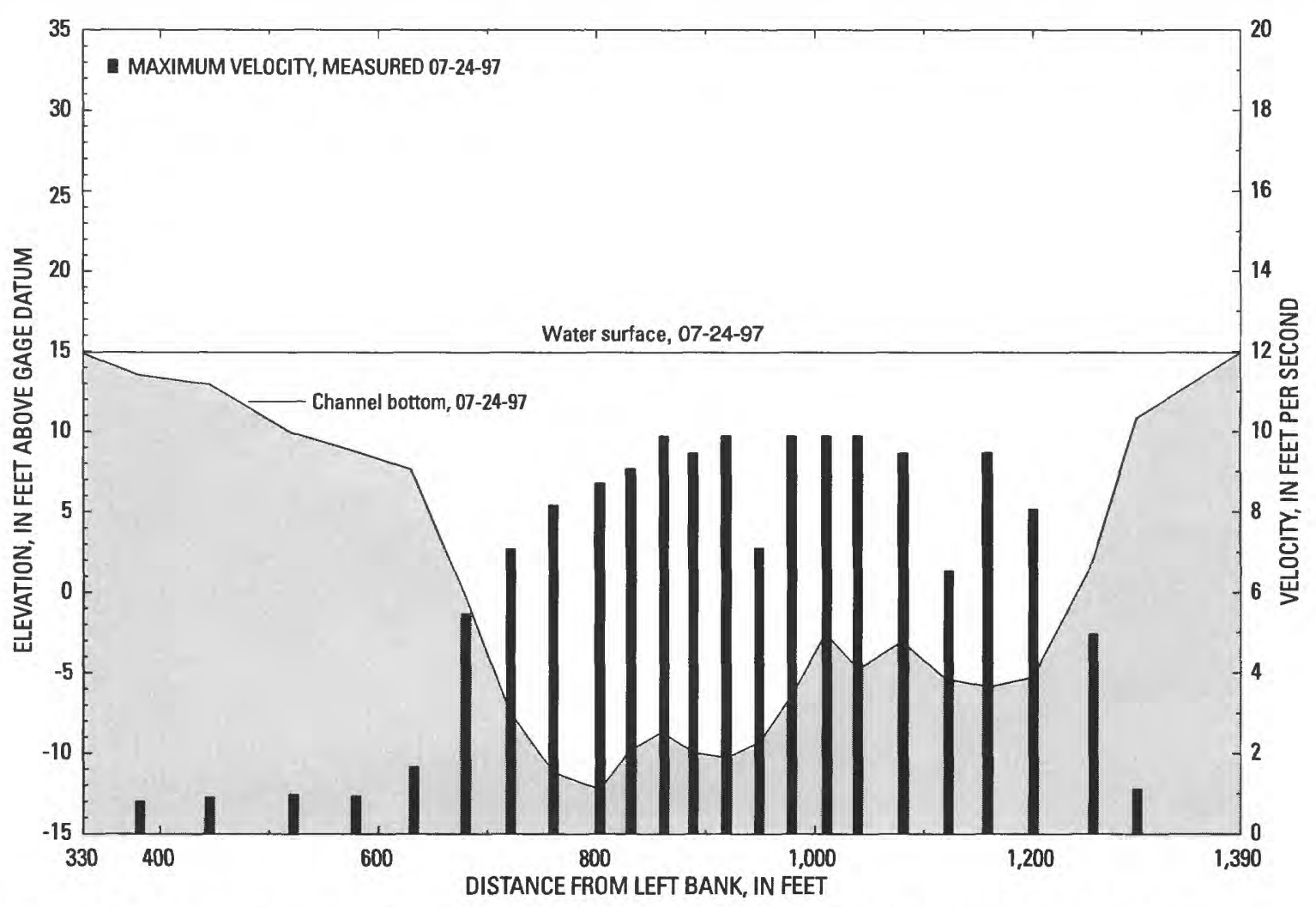

Figure 11. Channel cross section and maximum flow velocity at station 02129000 Pee Dee River near Rockingham, N.C. 
DISCHARGE MEASUREMENT DATA FOR STATION 02129000 PEE DEE RIVER NEAR ROCKINGHAM, N.C.

[ft, foot; ft/s, foot per second]

\begin{tabular}{|c|c|c|}
\hline \multicolumn{3}{|c|}{ Discharge measurement date, 07-24-97 } \\
\hline $\begin{array}{l}\text { Distance from } \\
\text { starting point on } \\
\text { left bank } \\
\text { (ft) }\end{array}$ & $\begin{array}{c}\text { Depth } \\
\text { (ft) }\end{array}$ & $\begin{array}{l}\text { Maximum } \\
\text { velocity } \\
(\mathrm{ft} / \mathrm{s})\end{array}$ \\
\hline 325.00 & 0 & 0.00 \\
\hline 380.00 & 1.4 & 0.80 \\
\hline 445.00 & 2 & 0.89 \\
\hline 520.00 & 5 & 0.97 \\
\hline 580.00 & 6.2 & 0.93 \\
\hline 630.00 & 7.3 & 1.66 \\
\hline 680.00 & 15.2 & 5.46 \\
\hline 720.00 & 22.3 & 7.11 \\
\hline 760.00 & 26.1 & 8.17 \\
\hline 803.00 & 27.2 & 8.71 \\
\hline 830.00 & 24.8 & 9.07 \\
\hline 860.00 & 23.6 & 9.89 \\
\hline 890.00 & 24.9 & 9.46 \\
\hline 920.00 & 25.2 & 9.89 \\
\hline 950.00 & 24.2 & 7.11 \\
\hline 980.00 & 21.2 & 9.89 \\
\hline $1,010.00$ & 17.5 & 9.89 \\
\hline $1,040.00$ & 19.7 & 9.89 \\
\hline $1,080.00$ & 18 & 9.46 \\
\hline $1,120.00$ & 20.3 & 6.54 \\
\hline $1,160.00$ & 20.8 & 9.46 \\
\hline $1,200.00$ & 20.2 & 8.07 \\
\hline $1,255.00$ & 13 & 4.96 \\
\hline $1,295.00$ & 4.1 & 1.11 \\
\hline $1,390.00$ & 0 & 0.00 \\
\hline
\end{tabular}




\section{DROWNING CREEK NEAR HOFFMAN, N.C.}

GAGE LOCATION.--Lat $35^{\circ} 03^{\prime} 38^{\prime \prime}$, long $79^{\circ} 29^{\prime} 39^{\prime \prime}$, Richmond County, Hydrologic Unit 03040203 , on right bank $10 \mathrm{ft}$ downstream of bridge on U.S. Highway 1, 1 mi upstream from Seaboard Coast Line Railroad bridge, $0.8 \mathrm{mi}$ downstream of Deep Creek, and 4 mi northeast of Hoffman.

MEASUREMENT LOCATION.--Downstream side of bridge on U.S. Highway 1.

DRAINAGE AREA.--183 $\mathrm{mi}^{2}$.

PERIOD OF RECORD.--October 1939 to current year.

GAGE.--Water-stage recorder. Elevation of gage is $270 \mathrm{ft}$ above sea level, from topographic map. Satellite telemetry at station.

\section{DISCHARGE MEASUREMENT SUMMARY}

[ft/s, foot per second; $\mathrm{ft}$, foot; $\mathrm{ft}^{3} / \mathrm{s}$, cubic foot per second]

\begin{tabular}{ccccccc}
\hline $\begin{array}{c}\text { Date } \\
\text { of } \\
\text { measurement }\end{array}$ & $\begin{array}{c}\text { Stream stage } \\
\text { (feet above } \\
\text { datum) }\end{array}$ & $\begin{array}{c}\text { Average } \\
\text { velocity } \\
(\mathbf{f t / s})\end{array}$ & $\begin{array}{c}\text { Maximum } \\
\text { veloclty } \\
(\mathbf{f t} / \mathbf{s})\end{array}$ & $\begin{array}{c}\text { Maximum } \\
\text { depth } \\
\text { (ft) }\end{array}$ & $\begin{array}{c}\text { Discharge } \\
\left(\mathbf{f t}^{3} / \mathbf{s}\right)\end{array}$ & $\begin{array}{c}\text { Recurrence } \\
\text { Interval } \\
(\text { years) }\end{array}$ \\
\hline $09-01-64$ & 7.85 & 2.53 & 4.42 & 10.1 & 2,860 & $5-10$ \\
$09-07-96$ & 7.02 & 2.38 & 4.07 & 8.3 & 1,900 & $2-5$ \\
\hline
\end{tabular}

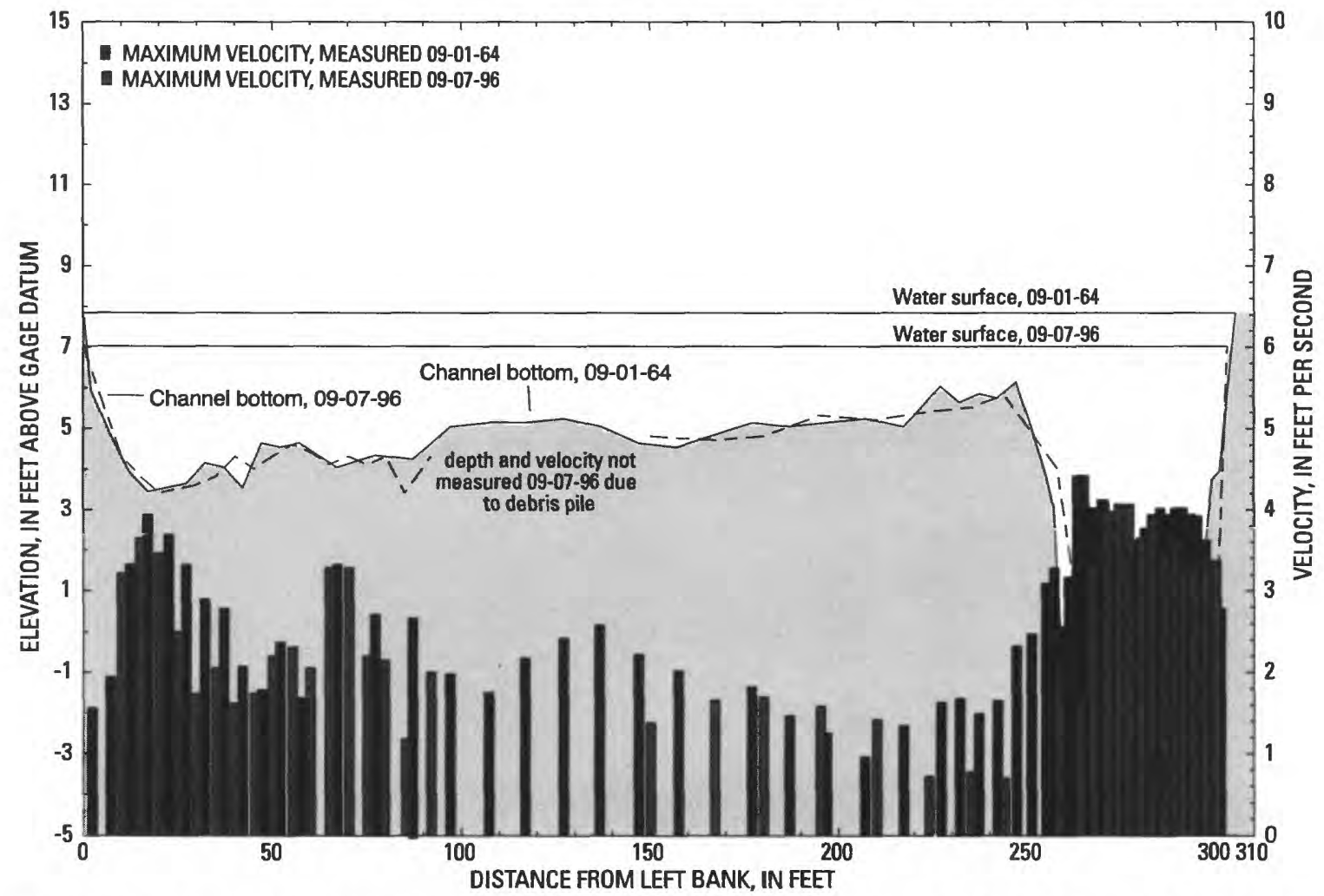

Figure 12. Channel cross sections and maximum flow velocities at station 02133500 Drowning Creek near Hoffman, N.C. 
DISCHARGE MEASUREMENT DATA FOR STATION 02133500 DROWNING CREEK NEAR HOFFMAN, N.C.

[ft, foot; ft/s, foot per second; nd, no data]

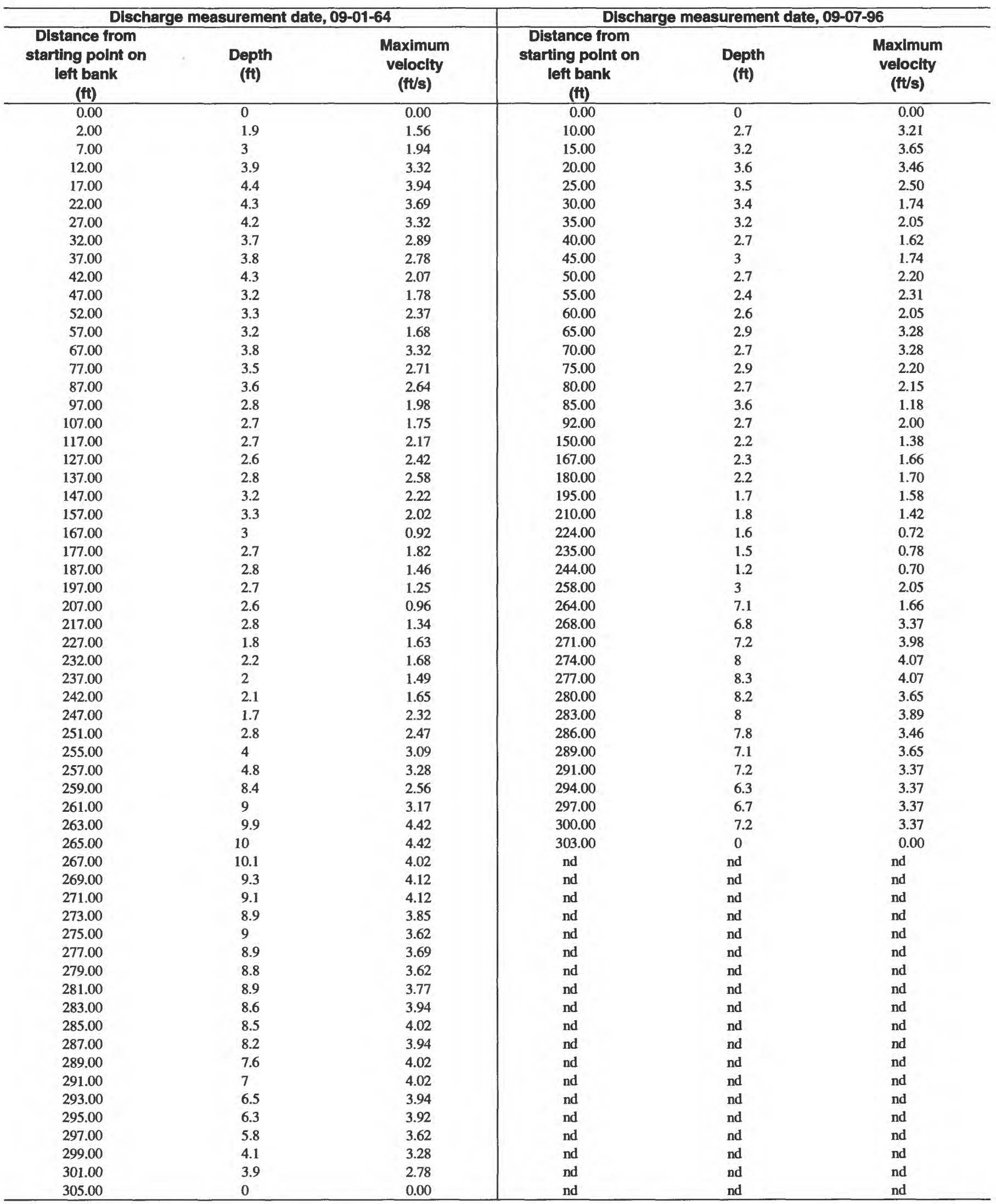




\section{LUMBER RIVER NEAR MAXTON, N.C.}

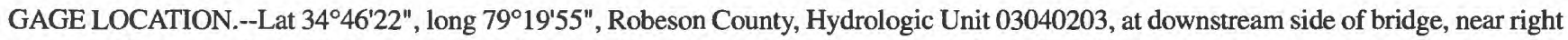
center of span, on State Highway 71, 2.6 mi north of Maxton, and 7.5 mi upstream from Gum Swamp.

MEASUREMENT LOCATION.--Upstream side of bridge on State Highway 71.

DRAINAGE AREA.--365 $\mathrm{mi}^{2}$.

PERIOD OF RECORD.--Occasional discharge measurements, water years 1974, 1980-85. June 1987 to current year.

GAGE.--Water-stage recorder with satellite telemetry. Elevation of gage is $180 \mathrm{ft}$ above sea level, from topographic map.

\section{DISCHARGE MEASUREMENT SUMMARY}

[ft/s, foot per second; $\mathrm{ft}$, foot; $\mathrm{ft}^{3} / \mathrm{s}$, cubic foot per second]

\begin{tabular}{ccccccc}
\hline $\begin{array}{c}\text { Date } \\
\text { of } \\
\text { measurement }\end{array}$ & $\begin{array}{c}\text { Stream stage } \\
\text { (feet above } \\
\text { datum) }\end{array}$ & $\begin{array}{c}\text { Average } \\
\text { velocity } \\
(\mathrm{ft} / \mathrm{s})\end{array}$ & $\begin{array}{c}\text { Maximum } \\
\text { velocity } \\
(\mathrm{ft} / \mathrm{s})\end{array}$ & $\begin{array}{c}\text { Maximum } \\
\text { depth } \\
(\mathrm{ft})\end{array}$ & $\begin{array}{c}\text { Discharge } \\
\left(\mathrm{ft}^{3} / \mathrm{s}\right)\end{array}$ & $\begin{array}{c}\text { Recurrence } \\
\text { interval } \\
(\text { years) }\end{array}$ \\
\hline $09-10-96$ & 12.87 & 1.95 & 3.13 & 12.7 & 2,520 & $5-10$ \\
\hline
\end{tabular}

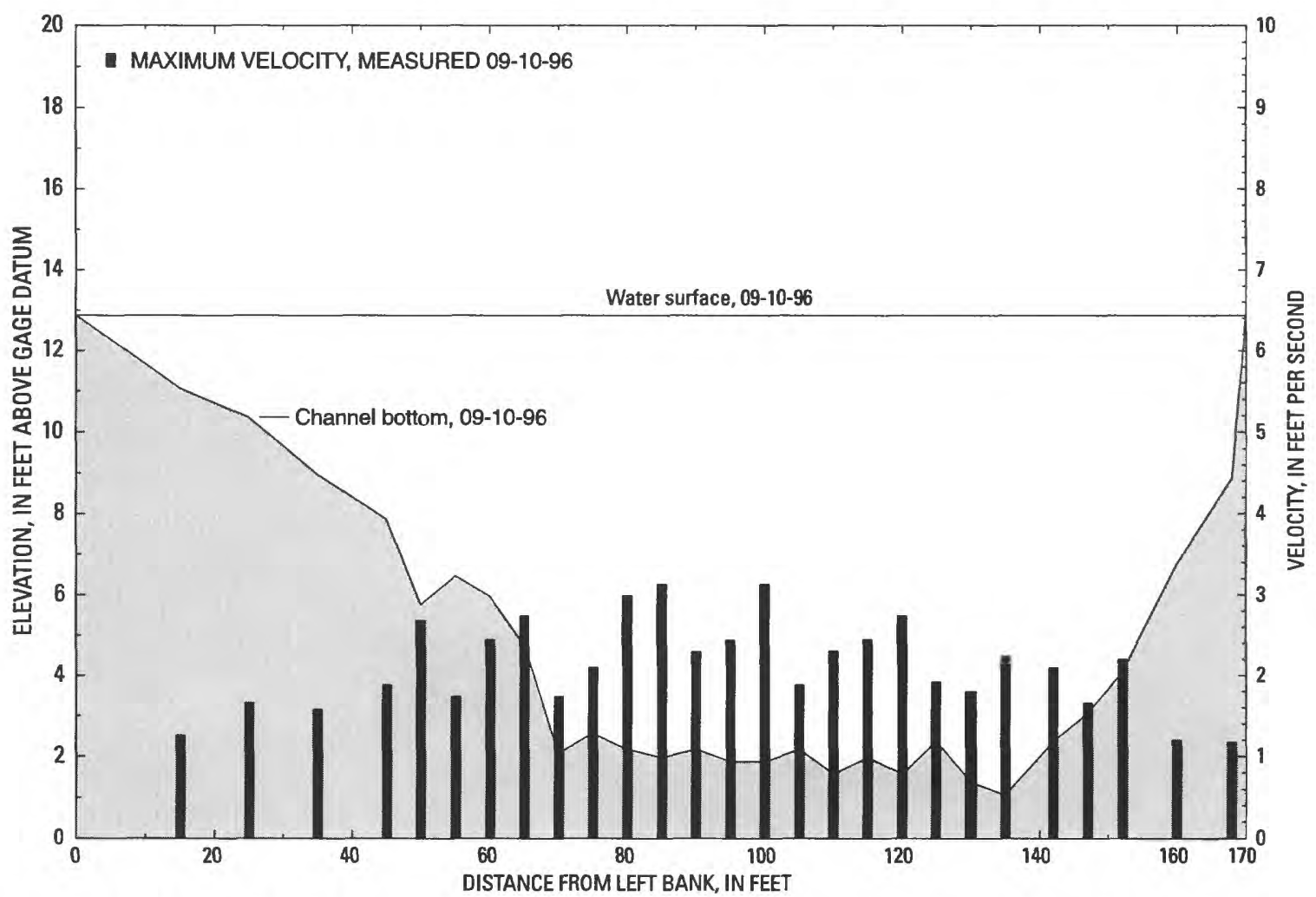

Figure 13. Channel cross section and maximum flow velocity at station 02133624 Lumber River near Maxton, N.C. 
DISCHARGE MEASUREMENT DATA FOR STATION 02133624 LUMBER RIVER NEAR MAXTON, N.C.

[ft, foot; ft/s, foot per second]

\begin{tabular}{|c|c|c|}
\hline \multicolumn{3}{|c|}{ Discharge measurement date, 09-10-96 } \\
\hline $\begin{array}{l}\text { Distance from } \\
\text { starting point on } \\
\text { left bank } \\
\text { (ft) }\end{array}$ & $\begin{array}{c}\text { Depth } \\
\text { (ft) }\end{array}$ & $\begin{array}{c}\text { Maximum } \\
\text { velocity } \\
\text { (ft/s) }\end{array}$ \\
\hline 0.00 & 0 & 0.00 \\
\hline 15.00 & 1.8 & 1.26 \\
\hline 25.00 & 2.5 & 1.66 \\
\hline 35.00 & 3.9 & 1.58 \\
\hline 45.00 & 5 & 1.88 \\
\hline 50.00 & 7.1 & 2.68 \\
\hline 55.00 & 6.4 & 1.74 \\
\hline 60.00 & 6.9 & 2.44 \\
\hline 65.00 & 8.1 & 2.74 \\
\hline 70.00 & 10.8 & 1.74 \\
\hline 75.00 & 10.3 & 2.10 \\
\hline 80.00 & 10.7 & 2.99 \\
\hline 85.00 & 10.9 & 3.13 \\
\hline 90.00 & 10.7 & 2.29 \\
\hline 95.00 & 11 & 2.44 \\
\hline 100.00 & 11 & 3.13 \\
\hline 105.00 & 10.7 & 1.88 \\
\hline 110.00 & 11.3 & 2.31 \\
\hline 115.00 & 10.9 & 2.44 \\
\hline 120.00 & 11.3 & 2.74 \\
\hline 125.00 & 10.5 & 1.92 \\
\hline 130.00 & 11.5 & 1.80 \\
\hline 135.00 & 11.8 & 2.24 \\
\hline 142.00 & 10.5 & 2.10 \\
\hline 147.00 & 9.8 & 1.66 \\
\hline 152.00 & 8.8 & 2.20 \\
\hline 160.00 & 6.1 & 1.20 \\
\hline 168.00 & 4 & 1.17 \\
\hline 170.00 & 0 & 0.00 \\
\hline
\end{tabular}




\section{CATAWBA RIVER NEAR PLEASANT GARDENS, NC}

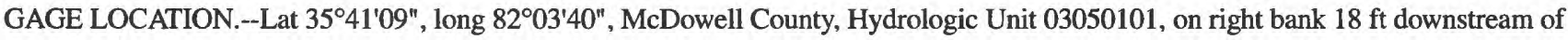
bridge on Secondary Road 1221, 0.8 mi upstream from Buck Creek, 0.8 mi southeast of Pleasant Gardens, and at river mile 297.

MEASUREMENT LOCATION.--Downstream side of bridge on Secondary Road 1221.

DRAINAGE AREA.- $126 \mathrm{mi}^{2}$.

PERIOD OF RECORD.--Occasional low-flow measurements, water years 1963, 1970-73, 1975. October 1980 to current year.

GAGE.--Water-stage recorder with satellite telemetry. Elevation of gage is $1,230 \mathrm{ft}$ above sea level, from topographic map. Telephone telemetry at station.

\section{DISCHARGE MEASUREMENT SUMMARY}

[ft/s, foot per second; $\mathrm{ft}$, foot; $\mathrm{ft}^{3} / \mathrm{s}$, cubic foot per second]

\begin{tabular}{ccccccc}
\hline $\begin{array}{c}\text { Date } \\
\text { of } \\
\text { measurement }\end{array}$ & $\begin{array}{c}\text { Stream stage } \\
\text { (feet above } \\
\text { datum) }\end{array}$ & $\begin{array}{c}\text { Average } \\
\text { velocity } \\
(\mathbf{f t} / \mathbf{s})\end{array}$ & $\begin{array}{c}\text { Maximum } \\
\text { velocity } \\
(\mathrm{ft} / \mathrm{s})\end{array}$ & $\begin{array}{c}\text { Maximum } \\
\text { depth } \\
(\mathrm{ft})\end{array}$ & $\begin{array}{c}\text { Dlscharge } \\
\left(\mathrm{ft}^{3} / \mathrm{s}\right)\end{array}$ & $\begin{array}{c}\text { Recurrence } \\
\text { Interval } \\
(\text { years) }\end{array}$ \\
\hline $08-27-95$ & 12.18 & 4.81 & 9.67 & 15.1 & 9,100 & 10 \\
\hline
\end{tabular}

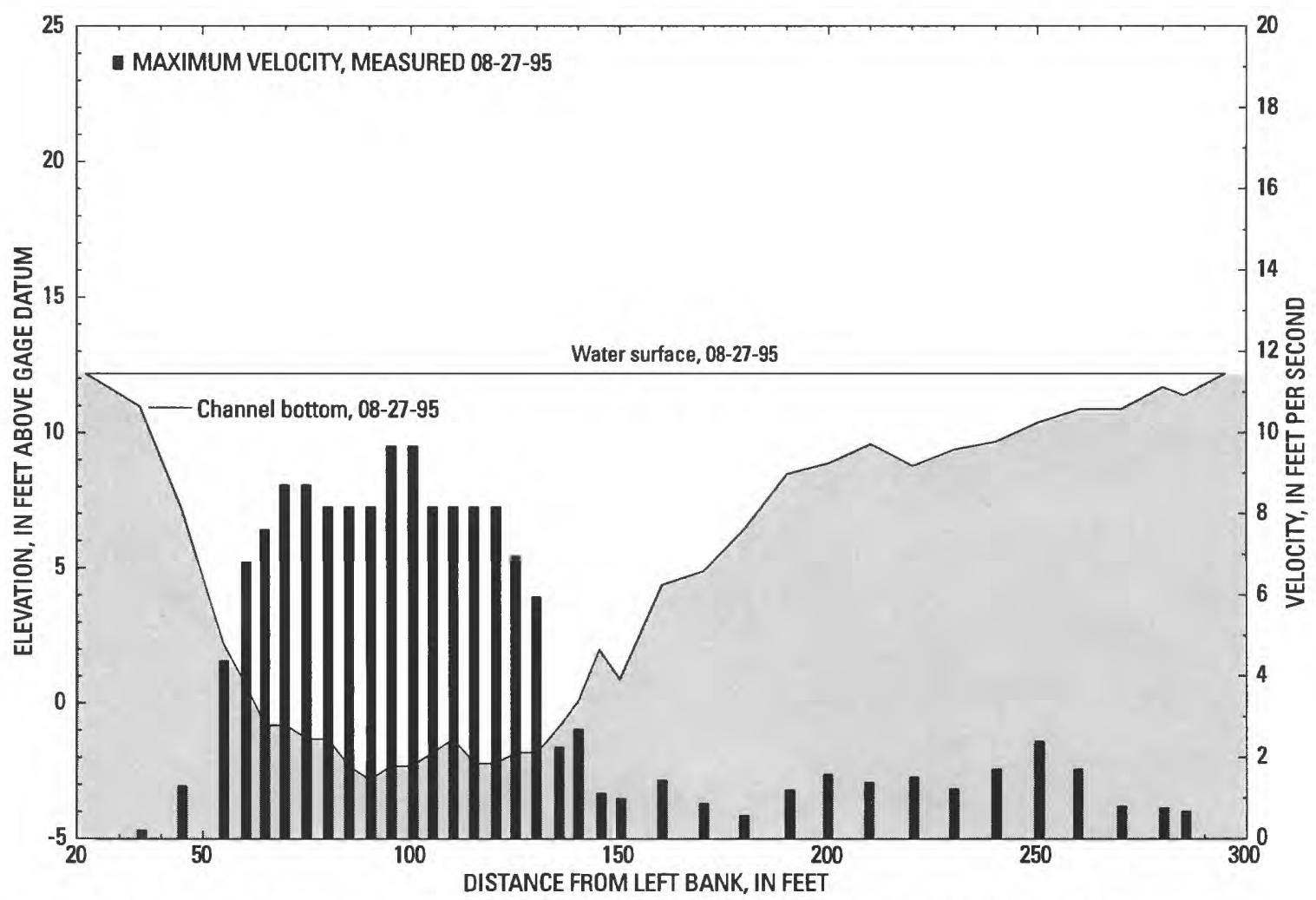

Figure 14. Channel cross section and maximum flow velocity at station 02137727 Catawba River near Pleasant Gardens, N.C. 
DISCHARGE MEASUREMENT DATA FOR STATION 02137727

CATAWBA RIVER NEAR PLEASANT GARDENS, N.C.

[ft, foot; ft/s, foot per second]

\begin{tabular}{|c|c|c|}
\hline \multicolumn{3}{|c|}{ Discharge measurement date, 08-27-95 } \\
\hline $\begin{array}{l}\text { Distance from } \\
\text { starting point on } \\
\text { left bank } \\
\text { (tt) }\end{array}$ & $\begin{array}{c}\text { Depth } \\
\text { (ft) }\end{array}$ & $\begin{array}{c}\text { Maximum } \\
\text { velocity } \\
\text { (ft/s) }\end{array}$ \\
\hline 22.00 & 0 & 0.00 \\
\hline 35.00 & 1.2 & 0.20 \\
\hline 45.00 & 5 & 1.29 \\
\hline 55.00 & 10 & 4.37 \\
\hline 60.00 & 11.4 & 6.81 \\
\hline 65.00 & 13 & 7.60 \\
\hline 70.00 & 13 & 8.71 \\
\hline 75.00 & 13.5 & 8.71 \\
\hline 80.00 & 13.5 & 8.17 \\
\hline 85.00 & 14.5 & 8.17 \\
\hline 90.00 & 15 & 8.17 \\
\hline 95.00 & 14.5 & 9.67 \\
\hline 100.00 & 14.5 & 9.67 \\
\hline 105.00 & 14 & 8.17 \\
\hline 110.00 & 13.5 & 8.17 \\
\hline 115.00 & 14.4 & 8.17 \\
\hline 120.00 & 14.4 & 8.17 \\
\hline 125.00 & 14 & 6.96 \\
\hline 130.00 & 14 & 5.95 \\
\hline 135.00 & 13.1 & 2.25 \\
\hline 140.00 & 12.1 & 2.68 \\
\hline 145.00 & 10.2 & 1.11 \\
\hline 150.00 & 11.3 & 0.99 \\
\hline 160.00 & 7.8 & 1.44 \\
\hline 170.00 & 7.3 & 0.86 \\
\hline 180.00 & 5.7 & 0.57 \\
\hline 190.00 & 3.7 & 1.20 \\
\hline 200.00 & 3.3 & 1.58 \\
\hline 210.00 & 2.6 & 1.38 \\
\hline 220.00 & 3.4 & 1.51 \\
\hline 230.00 & 2.8 & 1.23 \\
\hline 240.00 & 2.5 & 1.70 \\
\hline 250.00 & 1.8 & 2.39 \\
\hline 260.00 & 1.3 & 1.70 \\
\hline 270.00 & 1.3 & 0.80 \\
\hline 280.00 & 0.5 & 0.75 \\
\hline 285.00 & 0.8 & 0.67 \\
\hline 295.00 & 0 & 0.00 \\
\hline
\end{tabular}




\section{HENRY FORK NEAR HENRY RIVER, N.C.}

GAGE LOCATION.--Lat $35^{\circ} 41^{\prime} 03^{\prime \prime}$, long $81^{\circ} 24^{\prime} 10^{\prime \prime}$, Catawba County, Hydrologic Unit 03050102 , on left bank $325 \mathrm{ft}$ downstream of bridge on Secondary Road 1124, at site of Old Link Ford, 1.2 mi downstream of Burke-Catawba County line, and 2 mi southeast of Henry River.

MEASUREMENT LOCATION.--Downstream side of bridge on Secondary Road 1124, $325 \mathrm{ft}$ upstream of gage.

DRAINAGE AREA.--83.2 $\mathrm{mi}^{2}$.

PERIOD OF RECORD.--July 1925 to November 1931, December 1941 to current year.

GAGE.--Water-stage recorder with satellite telemetry. Datum of gage is $891.0 \mathrm{ft}$ above sea level. July 1925 to November 1931 , at site $450 \mathrm{ft}$ upstream at same datum.

EXTREMES OUTSIDE PERIOD OF RECORD.--Maximum stage known: $29.2 \mathrm{ft}$, Aug. 13, 1940, at former site, from floodmarks; discharge: $31,300 \mathrm{ft}^{3} / \mathrm{s}$. The flood of July 16,1916 , reached a stage of about $23 \mathrm{ft}$ at former site; discharge: $20,700 \mathrm{ft}^{3} / \mathrm{s}$.

\section{DISCHARGE MEASUREMENT SUMMARY}

[ft/s, foot per second; $\mathrm{ft}$, foot; $\mathrm{ft}^{3} / \mathrm{s}$, cubic foot per second]

\begin{tabular}{ccccccc}
\hline $\begin{array}{c}\text { Date } \\
\text { of } \\
\text { measurement }\end{array}$ & $\begin{array}{c}\text { Stream stage } \\
\text { (feet above } \\
\text { datum) }\end{array}$ & $\begin{array}{c}\text { Average } \\
\text { velocity } \\
(\mathrm{ft} / \mathrm{s})\end{array}$ & $\begin{array}{c}\text { Maximum } \\
\text { velocity } \\
(\mathrm{ft} / \mathrm{s})\end{array}$ & $\begin{array}{c}\text { Maximum } \\
\text { depth } \\
(\mathrm{ft})\end{array}$ & $\begin{array}{c}\text { Discharge } \\
\left(\mathrm{ft} \mathrm{t}^{3} / \mathrm{s}\right)\end{array}$ & $\begin{array}{c}\text { Recurrence } \\
\text { interval } \\
(\text { years })\end{array}$ \\
\hline $08-17-94$ & 12.93 & 4.83 & 8.89 & 15.6 & 6,420 \\
\hline
\end{tabular}

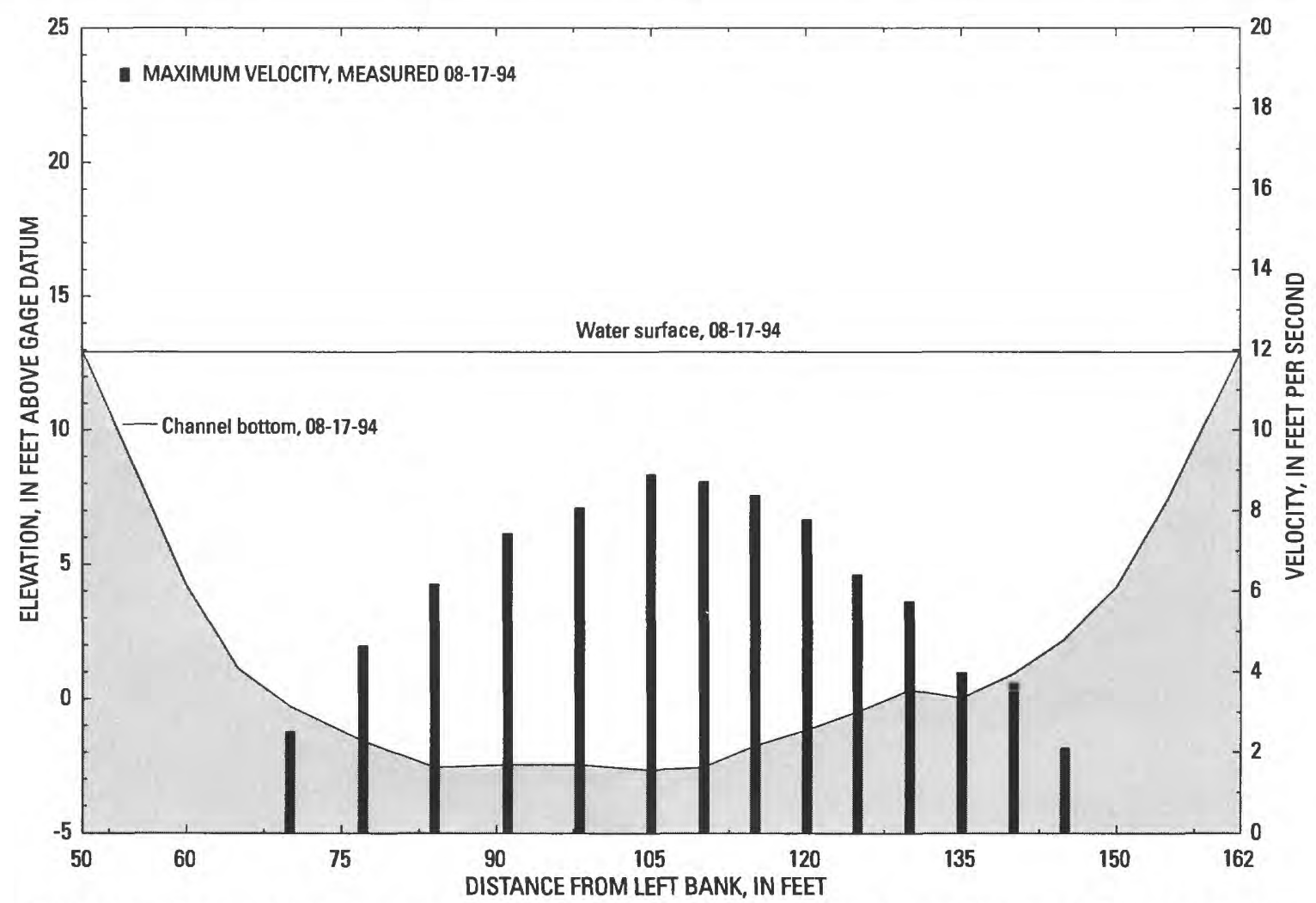

Figure 15. Channel cross section and maximum flow velocity at station 02143000 Henry Fork near Henry River, N.C. 
DISCHARGE MEASUREMENT DATA FOR STATION 021413000 HENRY FORK NEAR HENRY RIVER, N.C.

[ft, foot; ft/s, foot per second]

\begin{tabular}{|c|c|c|}
\hline \multicolumn{3}{|c|}{ Discharge measurement date, 08-17-94 } \\
\hline $\begin{array}{l}\text { Distance from } \\
\text { starting point on } \\
\text { left bank } \\
\text { (ft) }\end{array}$ & $\begin{array}{l}\text { Depth } \\
\text { (ft) }\end{array}$ & $\begin{array}{c}\text { Maximum } \\
\text { velocity } \\
(\mathrm{ft} / \mathrm{s})\end{array}$ \\
\hline 50.00 & 0 & 0.00 \\
\hline 60.00 & 8.7 & -0.24 \\
\hline 65.00 & 11.8 & -0.70 \\
\hline 70.00 & 13.2 & 2.50 \\
\hline 77.00 & 14.5 & 4.65 \\
\hline 84.00 & 15.5 & 6.17 \\
\hline 91.00 & 15.4 & 7.43 \\
\hline 98.00 & 15.4 & 8.07 \\
\hline 105.00 & 15.6 & 8.89 \\
\hline 110.00 & 15.5 & 8.71 \\
\hline 115.00 & 14.7 & 8.38 \\
\hline 120.00 & 14.1 & 7.78 \\
\hline 125.00 & 13.4 & 6.41 \\
\hline 130.00 & 12.6 & 5.74 \\
\hline 135.00 & 12.9 & 3.98 \\
\hline 140.00 & 12 & 3.72 \\
\hline 145.00 & 10.7 & 2.10 \\
\hline 150.00 & 8.8 & -0.55 \\
\hline 155.00 & 5.5 & -0.49 \\
\hline 162.00 & 0 & 0.00 \\
\hline
\end{tabular}




\section{TWELVE MILE CREEK NEAR WAXHAW, N.C.}

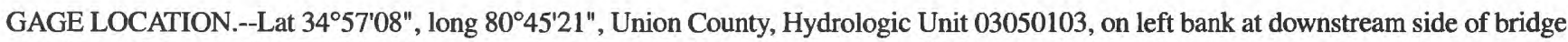
on State Highway 16, $680 \mathrm{ft}$ downstream of West Fork Twelve Mile Creek, and 2.5 mi north of Waxhaw.

MEASUREMENT LOCATION.--Downstream side of bridge on State Highway 16.

DRAINAGE AREA. - -76.5 $\mathrm{mi}^{2}$.

PERIOD OF RECORD.--Occasional low-flow measurements, water years 1949-60. October 1960 to current year.

GAGE.--Water-stage recorder. Datum of gage is $489.04 \mathrm{ft}$ above sea level. Prior to Mar. 13, 1962, water-stage recorder at site $20 \mathrm{ft}$ upstream, Mar. 13, 1962 to June 4, 1997, water-stage recorder at site 100 feet upstream at same datum. Telephone telemetry at station.

EXTREMES OUTSIDE PERIOD OF RECORD.--Maximum stage known since at least 1900 is $23.6 \mathrm{ft}$, Sept. 7, 1949, from floodmarks.

\section{DISCHARGE MEASUREMENT SUMMARY}

[ft/s, foot per second; $\mathrm{ft}$, foot; $\mathrm{ft}^{3} / \mathrm{s}$, cubic foot per second]

\begin{tabular}{ccccccc}
\hline $\begin{array}{c}\text { Date } \\
\text { of } \\
\text { measurement }\end{array}$ & $\begin{array}{c}\text { Stream stage } \\
\text { (feet above } \\
\text { datum) }\end{array}$ & $\begin{array}{c}\text { Average } \\
\text { velocity } \\
(\mathrm{ft} / \mathrm{s})\end{array}$ & $\begin{array}{c}\text { Maximum } \\
\text { velocity } \\
(\mathrm{ft} / \mathrm{s})\end{array}$ & $\begin{array}{c}\text { Maximum } \\
\text { depth } \\
(\mathrm{ft})\end{array}$ & $\begin{array}{c}\text { Discharge } \\
\left(\mathrm{ft}^{\mathbf{3}} / \mathbf{s}\right)\end{array}$ & $\begin{array}{c}\text { Recurrence } \\
\text { interval } \\
(\mathbf{y e a r s})\end{array}$ \\
\hline $08-27-95$ & 21.55 & 4.99 & 6.81 & 20.9 & 9,540 \\
\hline
\end{tabular}

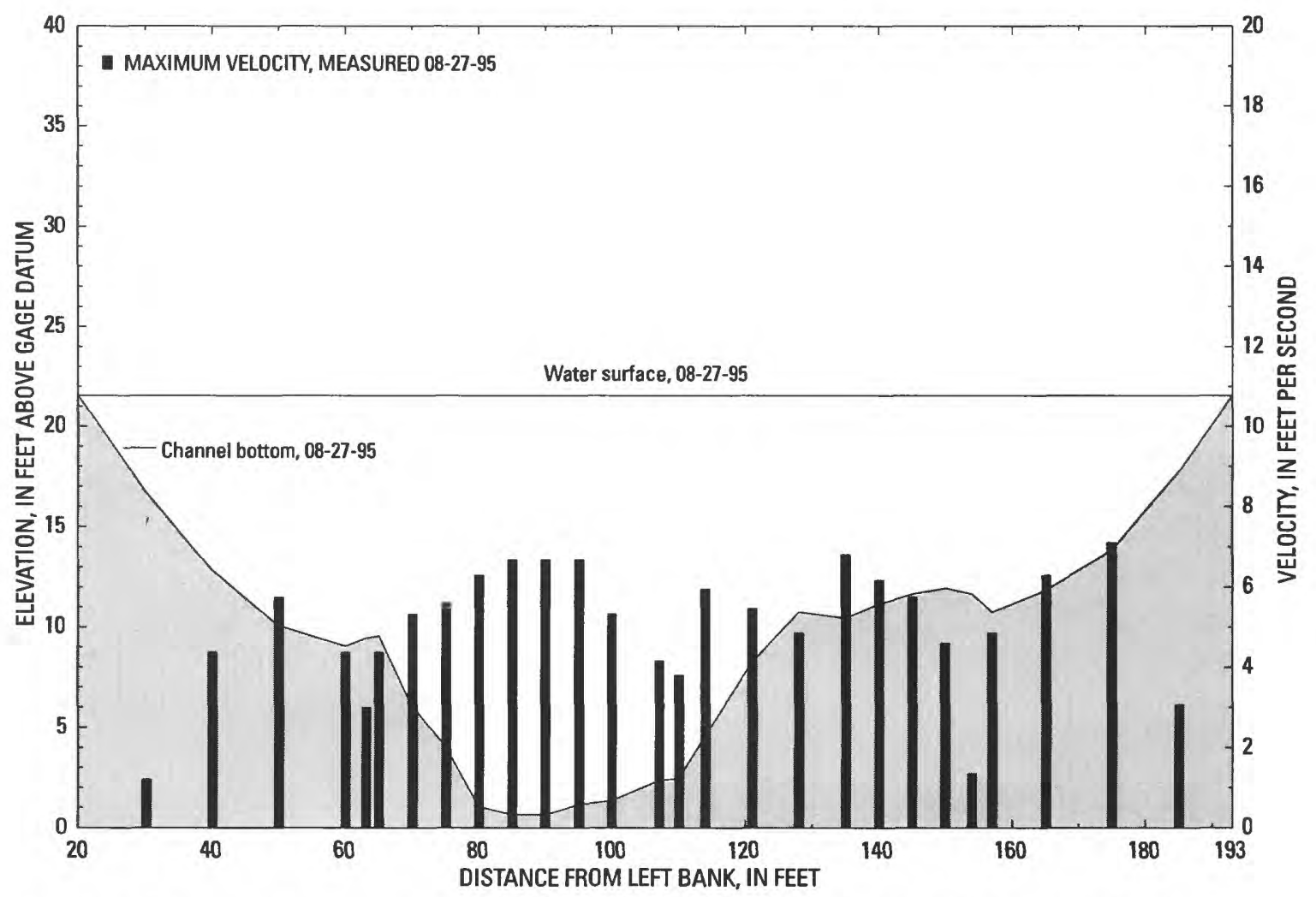

Figure 16. Channel cross section and maximum flow velocity at station 02146900 Twelve Mile Creek near Waxhaw, N.C. 
DISCHARGE MEASUREMENT DATA FOR STATION 02146900 TWELVE MILE CREEK NEAR WAXHAW, N.C.

[ft, foot; ft/s, foot per second]

\begin{tabular}{|c|c|c|}
\hline \multicolumn{3}{|c|}{ Discharge measurement date, 08-27-95 } \\
\hline $\begin{array}{l}\text { Distance from } \\
\text { starting point on } \\
\text { left bank } \\
\text { (ft) }\end{array}$ & $\begin{array}{l}\text { Depth } \\
\text { (ft) }\end{array}$ & $\begin{array}{c}\text { Maximum } \\
\text { velocity } \\
(\mathrm{ft} / \mathrm{s})\end{array}$ \\
\hline 20.00 & 0 & 0.00 \\
\hline 30.00 & 4.8 & 1.20 \\
\hline 40.00 & 8.7 & 4.37 \\
\hline 50.00 & 11.5 & 5.74 \\
\hline 60.00 & 12.5 & 4.37 \\
\hline 63.00 & 12.1 & 2.99 \\
\hline 65.00 & 12 & 4.37 \\
\hline 70.00 & 15.5 & 5.32 \\
\hline 75.00 & 17.5 & 5.60 \\
\hline 80.00 & 20.5 & 6.29 \\
\hline 85.00 & 20.9 & 6.67 \\
\hline 90.00 & 20.9 & 6.67 \\
\hline 95.00 & 20.4 & 6.67 \\
\hline 100.00 & 20.2 & 5.32 \\
\hline 107.00 & 19.2 & 4.16 \\
\hline 110.00 & 19.1 & 3.80 \\
\hline 114.00 & 16.9 & 5.95 \\
\hline 121.00 & 13.3 & 5.46 \\
\hline 128.00 & 10.8 & 4.85 \\
\hline 135.00 & 11.1 & 6.81 \\
\hline 140.00 & 10.4 & 6.17 \\
\hline 145.00 & 9.9 & 5.74 \\
\hline 150.00 & 9.6 & 4.59 \\
\hline 154.00 & 9.9 & 1.35 \\
\hline 157.00 & 10.8 & 4.85 \\
\hline 165.00 & 9.7 & 6.29 \\
\hline 175.00 & 7.7 & 7.11 \\
\hline 185.00 & 3.8 & 3.06 \\
\hline 193.00 & 0 & 0.00 \\
\hline
\end{tabular}




\section{COVE CREEK NEAR LAKE LURE, N.C.}

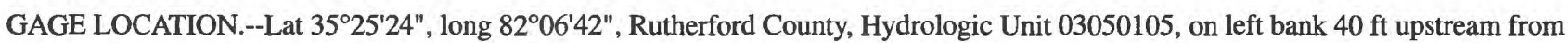
bridge on U.S. Highways 64 and 74, 1.0 mi upstream from mouth, and 5.0 mi east of town of Lake Lure.

MEASUREMENT LOCATION.--Upstream side of bridge on U.S. Highways 64 and 74, $40 \mathrm{ft}$ downstream of gage.

DRAINAGE AREA.--79.0 $\mathrm{mi}^{2}$

PERIOD OF RECORD.--Occasional low-flow measurements, water years 1949-50. October 1950 to current year. Monthly discharge only for some periods, published in WSP 1723.

GAGE.--Water-stage recorder with satellite telemetry. Datum of gage is $815.4 \mathrm{ft}$ above sea level. Prior to Dec. 20, 1954, nonrecording gage at same site and datum.

EXTREMES OUTSIDE PERIOD OF RECORD.--Flood of 1916 reached a stage of about $23 \mathrm{ft}$, from records of North Carolina State Highway Commission.

\section{DISCHARGE MEASUREMENT SUMMARY}

[ft/s, foot per second; $\mathrm{ft}$, foot; $\mathrm{ft}^{3} / \mathrm{s}$, cubic foot per second]

\begin{tabular}{ccccccc}
\hline $\begin{array}{c}\text { Date } \\
\text { of } \\
\text { measurement }\end{array}$ & $\begin{array}{c}\text { Stream stage } \\
\text { (feet above } \\
\text { datum) }\end{array}$ & $\begin{array}{c}\text { Average } \\
\text { velocity } \\
(\mathrm{ft} / \mathrm{s})\end{array}$ & $\begin{array}{c}\text { Maximum } \\
\text { velocity } \\
(\mathrm{ft} / \mathrm{s})\end{array}$ & $\begin{array}{c}\text { Maximum } \\
\text { depth } \\
(\mathrm{ft})\end{array}$ & $\begin{array}{c}\text { Discharge } \\
\left(\mathrm{ft}^{\mathbf{3}} / \mathrm{s}\right)\end{array}$ & $\begin{array}{c}\text { Recurrence } \\
\text { interval } \\
(\mathrm{years})\end{array}$ \\
\hline $10-04-64$ & 15.45 & 4.31 & 6.15 & 16.7 & 4,530 \\
\hline
\end{tabular}

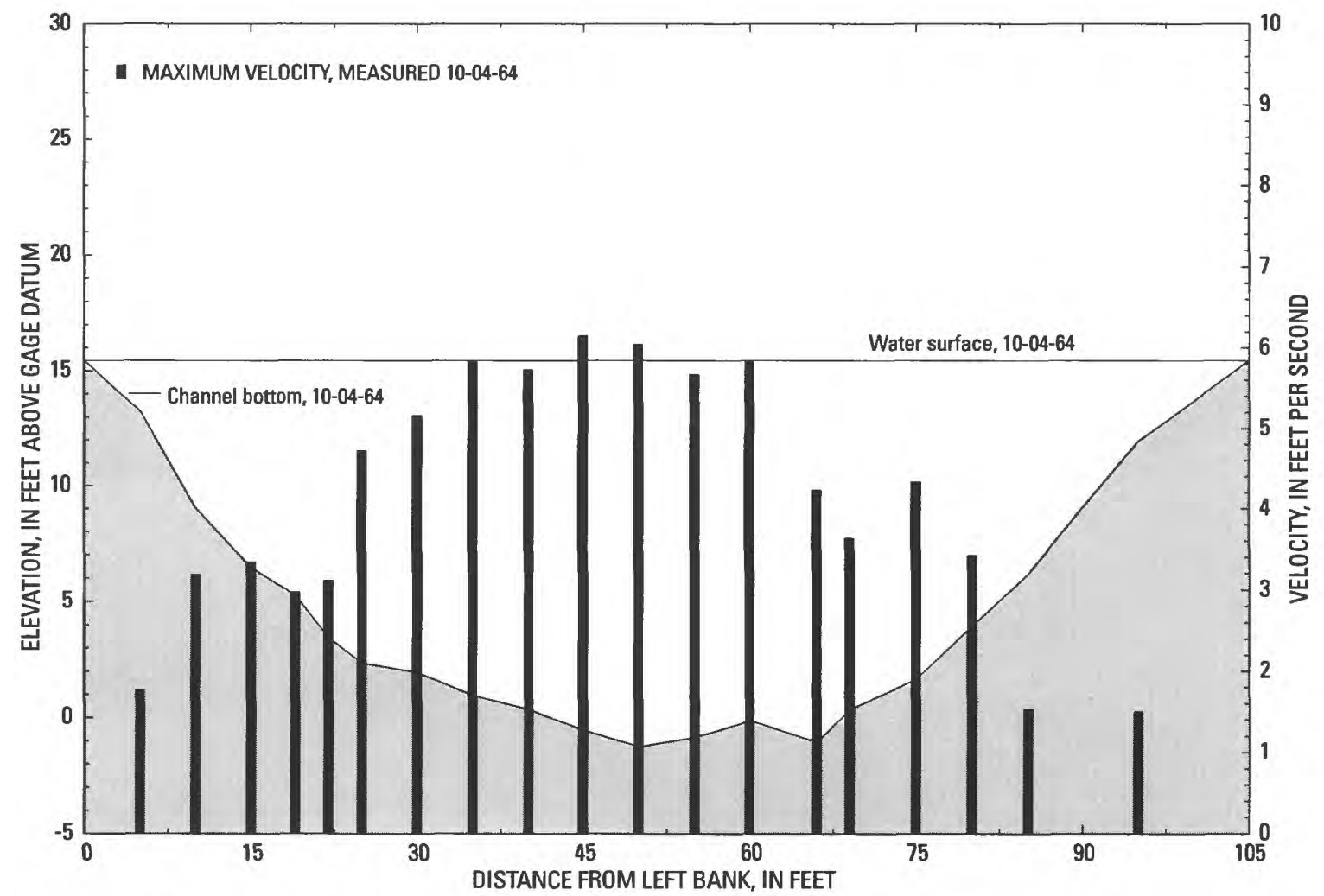

Figure 17. Channel cross section and maximum flow velocity at station 02149000 Cove Creek near Lake Lure, N.C. 
DISCHARGE MEASUREMENT DATA FOR STATION 02149000 COVE CREEK NEAR LAKE LURE, N.C.

[ft, foot; ft/s, foot per second]

\begin{tabular}{ccc}
\hline \multicolumn{2}{c}{ Discharge measurement date, 10-04-64 } \\
\hline $\begin{array}{c}\text { Distance from } \\
\text { starting point on } \\
\text { left bank } \\
\text { ( } \mathbf{t t})\end{array}$ & $\begin{array}{c}\text { Depth } \\
\text { ( } \mathbf{t} \text { ) }\end{array}$ & $\begin{array}{c}\text { Maximum } \\
\text { velocity } \\
\text { (ft/s) }\end{array}$ \\
\hline 0.00 & 0 & 0.00 \\
5.00 & 2.2 & 1.76 \\
10.00 & 6.4 & 3.18 \\
15.00 & 9 & 3.34 \\
19.00 & 10.2 & 2.97 \\
22.00 & 12 & 3.11 \\
25.00 & 13.1 & 4.72 \\
30.00 & 13.5 & 5.16 \\
35.00 & 14.5 & 5.83 \\
40.00 & 15.1 & 5.73 \\
45.00 & 16 & 6.15 \\
50.00 & 16.7 & 6.04 \\
55.00 & 16.3 & 5.67 \\
60.00 & 15.6 & 5.83 \\
66.00 & 16.5 & 4.23 \\
69.00 & 15.1 & 3.63 \\
75.00 & 13.8 & 4.33 \\
80.00 & 11.5 & 3.42 \\
85.00 & 9.3 & 1.53 \\
95.00 & 3.5 & 1.50 \\
105.00 & 0 & 0.00 \\
\hline
\end{tabular}




\section{FRENCH BROAD RIVER AT ROSMAN, N.C.}

GAGE LOCATION.--Lat $35^{\circ} 08^{\prime} 32^{\prime \prime}$, long 8249'28", Transylvania County, Hydrologic Unit 06010105, on left bank $50 \mathrm{ft}$ upstream from bridge on U.S. Highway 178 at Rosman, 1.0 mi upstream from East Fork, and at river mile 216.4.

MEASUREMENT LOCATION.--Upstream side of bridge on U.S. Highway 178.

DRAINAGE AREA.--67.9 $\mathrm{mi}^{2}$.

PERIOD OF RECORD.--May 1907 to June 1909, October 1935 to current year. Monthly discharge only for some periods published in WSP 1306.

GAGE.--Water-stage recorder. Datum of gage is $2,173.83 \mathrm{ft}$ above sea level. Prior to June 30,1909 , nonrecording gage at site $500 \mathrm{ft}$ downstream at different datum. Jan. 1, 1936, to July 6, 1937, nonrecording gage at present site and datum. Satellite telemetry at station.

National Weather Service telephone telemetry at station.

EXTREMES OUTSIDE PERIOD OF RECORD.--Flood of July 1916 reached a stage of $13.9 \mathrm{ft}$, from floodmarks.

\section{DISCHARGE MEASUREMENT SUMMARY}

[ft/s, foot per second; $\mathrm{ft}$, foot; $\mathrm{ft}^{3} / \mathrm{s}$, cubic foot per second]

\begin{tabular}{ccccccc}
\hline $\begin{array}{c}\text { Date } \\
\text { of } \\
\text { measurement }\end{array}$ & $\begin{array}{c}\text { Stream stage } \\
\text { (feet above } \\
\text { datum) }\end{array}$ & $\begin{array}{c}\text { Average } \\
\text { velocity } \\
(\mathrm{ft} / \mathrm{s})\end{array}$ & $\begin{array}{c}\text { Maximum } \\
\text { velocity } \\
(\mathrm{ft} / \mathrm{s})\end{array}$ & $\begin{array}{c}\text { Maximum } \\
\text { depth } \\
\text { (ft) }\end{array}$ & $\begin{array}{c}\text { Discharge } \\
\left(\mathrm{ft}^{3} / \mathbf{s}\right)\end{array}$ & $\begin{array}{c}\text { Recurrence } \\
\text { Interval } \\
(\text { years) }\end{array}$ \\
\hline $03-04-79$ & 12.70 & 7.26 & 12.70 & 14.4 & 8,790 & $10-25$ \\
$11-22-91$ & 8.52 & 5.03 & 8.17 & 10.6 & 3,830 & $1-2$ \\
\hline
\end{tabular}

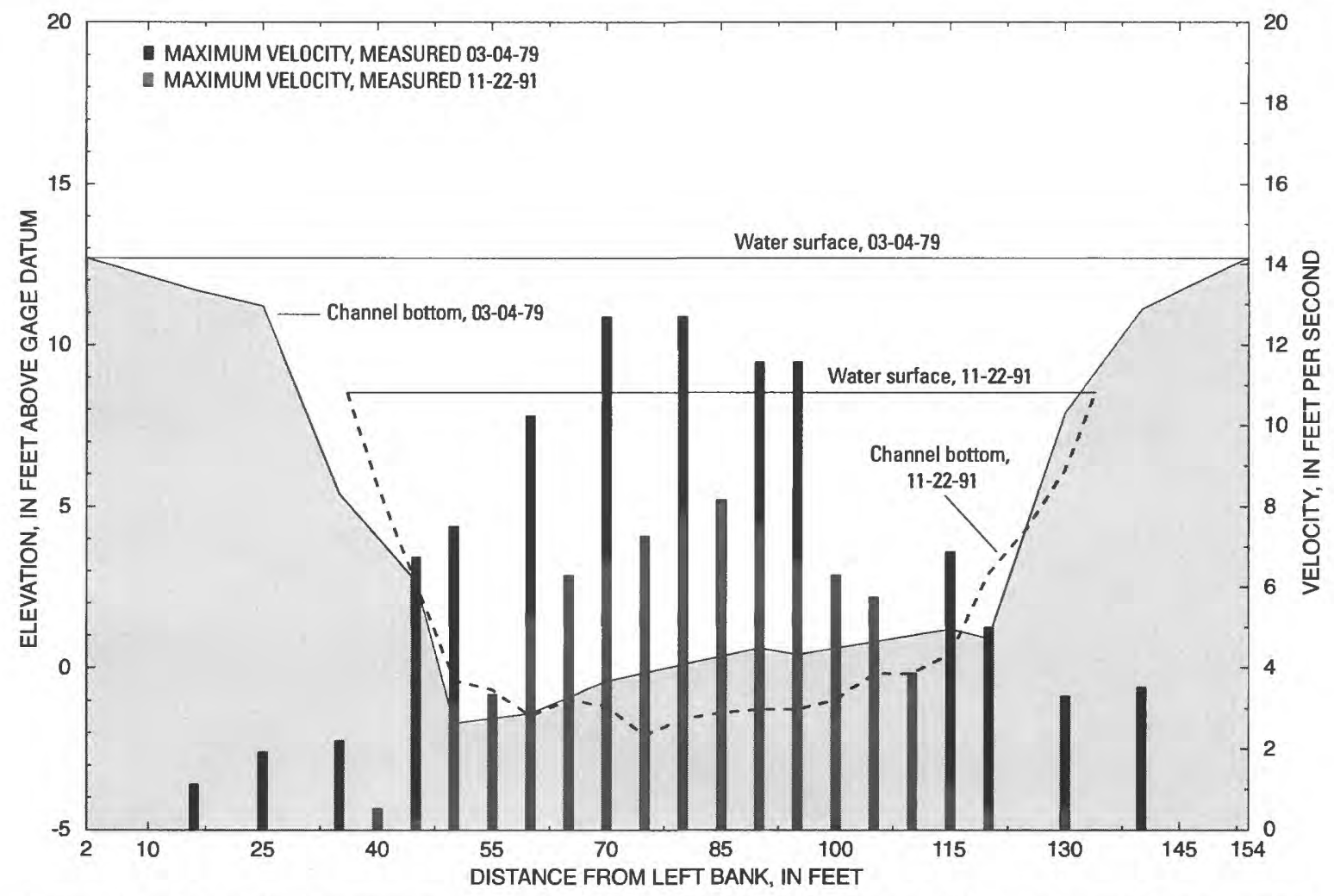

Figure 18. Channel cross sections and maximum flow velocities at station 03439000 French Broad River at Rosman, N.C. 
DISCHARGE MEASUREMENT DATA FOR STATION 03439000 FRENCH BROAD RIVER AT ROSMAN, N.C.

[ft, foot; ft/s, foot per second; nd, no data]

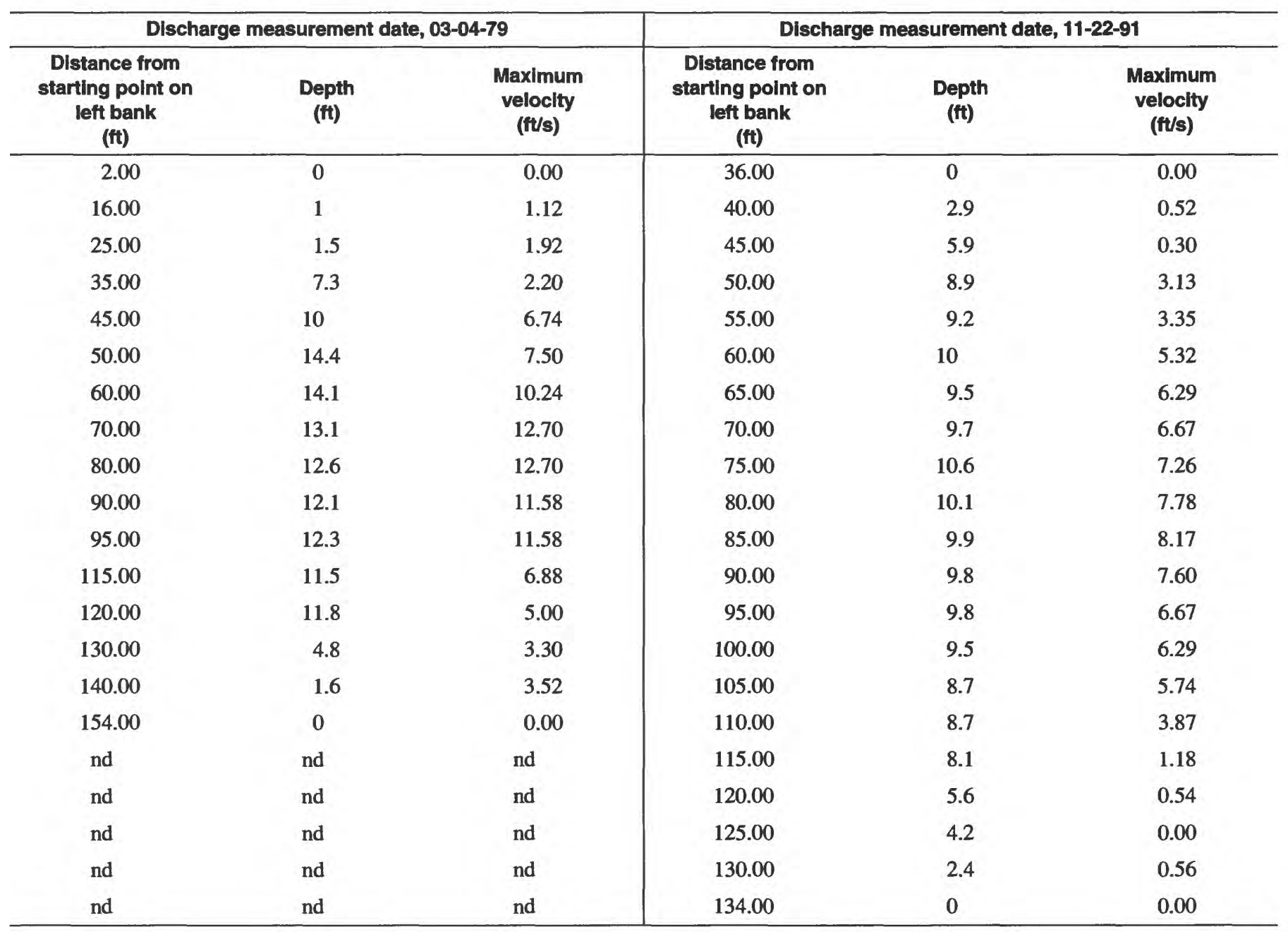




\section{FRENCH BROAD RIVER AT BLANTYRE, N.C.}

GAGE LOCATION.--Lat 35'17'56", long 82³7'26", Transylvania County, Hydrologic Unit 06010105, on left bank $40 \mathrm{ft}$ upstream from bridge on Secondary Road 1503, $700 \mathrm{ft}$ east of railroad at Blantyre, 3.5 mi downstream of Little River, and at river mile 183.7.

MEASUREMENT LOCATION.--Upstream side of bridge on Secondary Road 1503.

DRAINAGE AREA.--296 $\mathrm{mi}^{2}$.

PERIOD OF RECORD.--October 1920 to current year. Monthly discharge only for some periods, published in WSP 1306.

GAGE.--Water-stage recorder with satellite telemetry. Datum of gage is $2,060.32 \mathrm{ft}$ above sea level (levels by Tennessee Valley Authority). Prior to July 5, 1930, nonrecording gage at same site and datum. National Weather Service has telephone telemetry at station.

EXTREMES OUTSIDE PERIOD OF RECORD.--Since at least 1791, maximum stage $27.1 \mathrm{ft}$, July 16, 1916, from floodmarks (from studies by Tennessee Valley Authority).

\section{DISCHARGE MEASUREMENT SUMMARY}

[ft/s, foot per second; ft, foot; $\mathrm{ft}^{3} / \mathrm{s}$, cubic foot per second]

\begin{tabular}{ccccccc}
\hline $\begin{array}{c}\text { Date } \\
\text { of } \\
\text { measurement }\end{array}$ & $\begin{array}{c}\text { Stream stage } \\
\text { (feet above } \\
\text { datum) }\end{array}$ & $\begin{array}{c}\text { Average } \\
\text { velocity } \\
(\mathbf{f t} / \mathbf{s})\end{array}$ & $\begin{array}{c}\text { Maximum } \\
\text { velocity } \\
(\mathrm{ft} / \mathrm{s})\end{array}$ & $\begin{array}{c}\text { Maximum } \\
\text { depth } \\
\text { (ft) }\end{array}$ & $\begin{array}{c}\text { Discharge } \\
\text { (ft } \mathbf{3}^{3 / \mathrm{s})}\end{array}$ & $\begin{array}{c}\text { Recurrence } \\
\text { Interval } \\
(\text { years) }\end{array}$ \\
\hline $08-17-94$ & 22.37 & 3.59 & 6.70 & 23.5 & 13,000 & 10 \\
$01-09-98$ & 20.32 & 3.48 & 7.11 & 22.5 & 10,200 & 5 \\
\hline
\end{tabular}

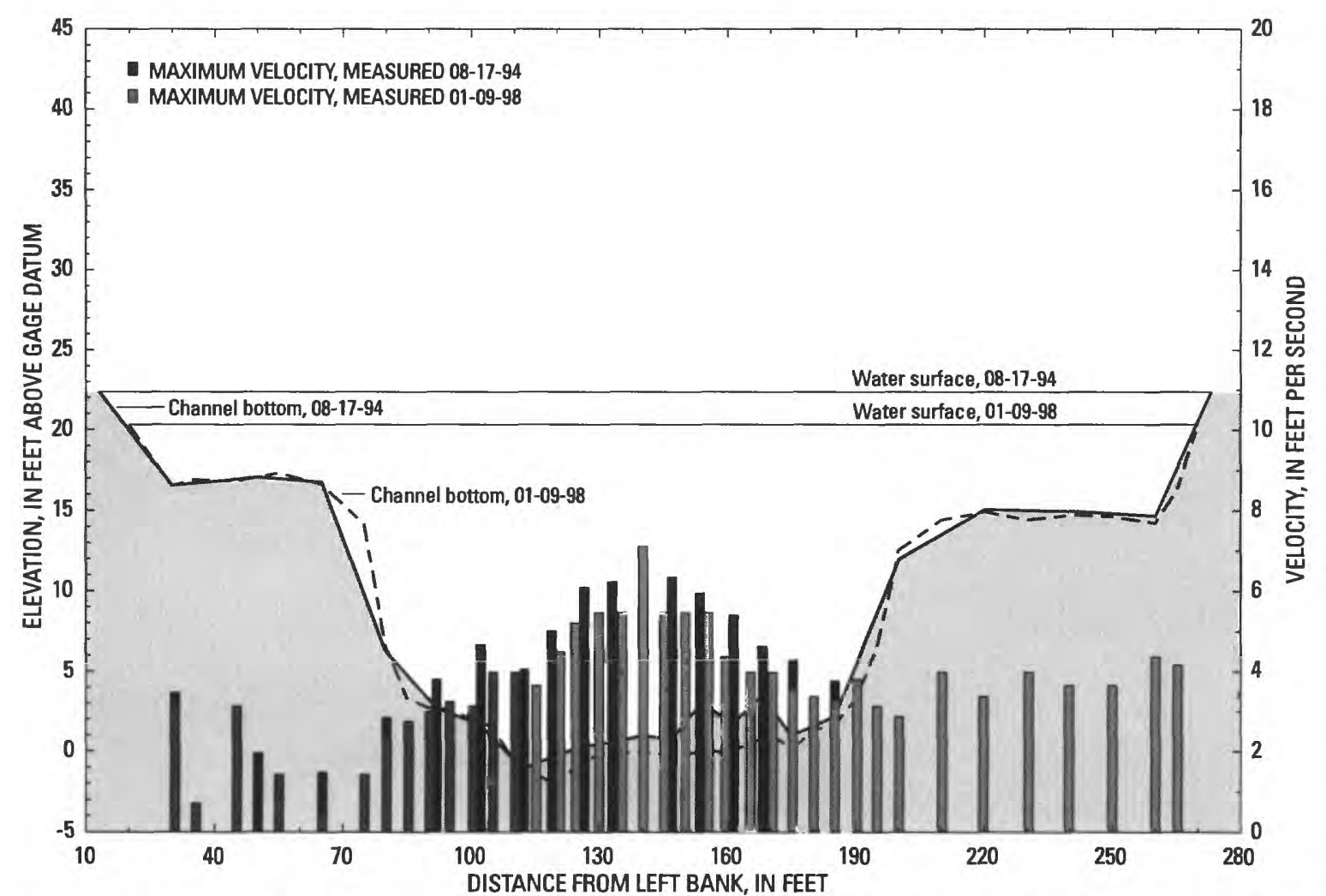

Figure 19. Channel cross sections and flow velocities at station 03443000 French Broad River at Blantyre, N.C. 
DISCHARGE MEASUREMENT DATA FOR STATION 03443000 FRENCH BROAD RIVER AT BLANTYRE, N.C.

[ft, foot; ft/s, foot per second; nd, no data]

\begin{tabular}{|c|c|c|c|c|c|}
\hline \multicolumn{3}{|c|}{ Discharge measurement date, 08-17-94 } & \multicolumn{3}{|c|}{ Discharge measurement date, 01-09-98 } \\
\hline $\begin{array}{l}\text { Distance from } \\
\text { starting point on } \\
\text { left bank } \\
\text { (ft) }\end{array}$ & $\begin{array}{l}\text { Depth } \\
\text { (ft) }\end{array}$ & $\begin{array}{l}\text { Maximum } \\
\text { veiocity } \\
\text { (ft/s) }\end{array}$ & $\begin{array}{l}\text { Distance from } \\
\text { starting point on } \\
\text { ieft bank } \\
\text { (ft) }\end{array}$ & $\begin{array}{l}\text { Depth } \\
\text { (ft) }\end{array}$ & $\begin{array}{c}\text { Maximum } \\
\text { velocity } \\
(\mathrm{ft} / \mathrm{s})\end{array}$ \\
\hline 13.00 & 0 & 0.00 & 20.00 & 0 & 0.00 \\
\hline 30.00 & 5.8 & 3.46 & 30.00 & 3.8 & 3.13 \\
\hline 50.00 & 5.3 & 1.94 & 35.00 & 3.4 & 0.70 \\
\hline 80.00 & 16.1 & 2.84 & 55.00 & 3 & 1.41 \\
\hline 92.00 & 19.7 & 3.80 & 65.00 & 3.7 & 0.42 \\
\hline 102.00 & 20.7 & 4.66 & 75.00 & 6.2 & 1.41 \\
\hline 112.00 & 23.5 & 4.05 & 80.00 & 13.8 & 2.39 \\
\hline 119.00 & 22.7 & 5.01 & 85.00 & 17 & 2.74 \\
\hline 147.00 & 21.6 & 6.34 & 105.00 & 18.8 & 3.98 \\
\hline 154.00 & 19.3 & 5.94 & 110.00 & 21 & 3.98 \\
\hline 161.00 & 20.7 & 5.40 & 115.00 & 21.7 & 3.65 \\
\hline 168.00 & 18.9 & 4.63 & 120.00 & 22.5 & 4.48 \\
\hline 175.00 & 21.4 & 4.28 & 125.00 & 21.1 & 5.20 \\
\hline 185.00 & 20.2 & 3.76 & 130.00 & 20.5 & 5.46 \\
\hline 200.00 & 10.4 & 2.32 & 135.00 & 20.4 & 5.46 \\
\hline 220.00 & 7.3 & 2.60 & 140.00 & 20.4 & 7.11 \\
\hline 240.00 & 7.4 & 3.06 & 145.00 & 20.5 & 5.46 \\
\hline 260.00 & 7.7 & 4.10 & 150.00 & 20.5 & 5.46 \\
\hline nd & nd & nd & 185.00 & 18.7 & 3.28 \\
\hline nd & nd & nd & 190.00 & 17 & 3.80 \\
\hline nd & nd & nd & 195.00 & 13.7 & 3.13 \\
\hline nd & nd & nd & 200.00 & 7.8 & 2.86 \\
\hline nd & nd & nd & 210.00 & 5.9 & 3.98 \\
\hline nd & nd & nd & 220.00 & 5.4 & 3.37 \\
\hline nd & nd & nd & 230.00 & 5.9 & 3.98 \\
\hline nd & nd & nd & 240.00 & 5.6 & 3.65 \\
\hline nd & nd & nd & 250.00 & 5.7 & 3.65 \\
\hline nd & nd & nd & 260.00 & 6.1 & 4.37 \\
\hline nd & nd & nd & 265.00 & 4.1 & 4.16 \\
\hline nd & nd & nd & 270.00 & 0.0 & 0.0 \\
\hline
\end{tabular}




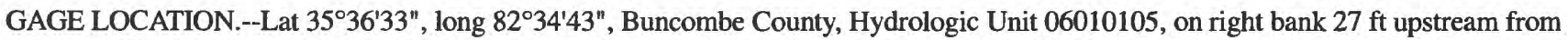
Pearson Bridge (Secondary Road 1348) at Asheville, 1.4 mi downstream of bridge on U.S. Highways 19 and 23, $3.2 \mathrm{mi}$ downstream of Swannanoa River, and at mile 145.8.

MEASUREMENT LOCATION.--Upstream side of Pearson Bridge (Secondary Road 1348).

DRAINAGE AREA.--945 $\mathrm{mi}^{2}$.

PERIOD OF RECORD.--October 1895 to current year. Monthly discharge only for some periods, published in WSP 1306.

GAGE.--Water-stage recorder. Datum of gage is $1,950.28 \mathrm{ft}$ above sea level. Sept. 17, 1895 , to Dec. 31, 1901, nonrecording gage at present site at different datum. Mar. 19, 1903, to July 15, 1916, and Jan. 1, 1917, to Sept. 30, 1922, nonrecording gage at Smith Bridge 1.5 mi upstream at datum $1961.80 \mathrm{ft}$. Oct. 1, 1922, to Aug. 9, 1930, nonrecording gage at present site and datum. Satellite telemetry at station. National Weather Service also has telephone telemetry at station.

EXTREMES OUTSIDE PERIOD OF RECORD.--Maximum stage observed since at least 1791 was that of July 16, 1916, and flood of June 17, 1876, reached a stage of $18 \mathrm{ft}$; from studies by Tennessee Valley Authority.

\section{DISCHARGE MEASUREMENT SUMMARY}

[ft/s, foot per second; $\mathrm{ft}$, foot; $\mathrm{ft}^{3} / \mathrm{s}$, cubic foot per second]

\begin{tabular}{ccccccc}
\hline $\begin{array}{c}\text { Date } \\
\text { of } \\
\text { measurement }\end{array}$ & $\begin{array}{c}\text { Stream stage } \\
\text { (feet above } \\
\text { datum) }\end{array}$ & $\begin{array}{c}\text { Average } \\
\text { velocity } \\
(\mathrm{ft} / \mathrm{s})\end{array}$ & $\begin{array}{c}\text { Maximum } \\
\text { velocity } \\
(\mathrm{ft} / \mathrm{s})\end{array}$ & $\begin{array}{c}\text { Maximum } \\
\text { depth } \\
(\mathrm{ft})\end{array}$ & $\begin{array}{c}\text { Discharge } \\
\left(\mathrm{ft}^{3} / \mathrm{s}\right)\end{array}$ & $\begin{array}{c}\text { Recurrence } \\
\text { interval } \\
(\text { (years) }\end{array}$ \\
\hline $11-06-77$ & 11.94 & 7.54 & 12.78 & 15.8 & 32,800 & 25 \\
$01-15-95$ & 10.69 & 6.75 & 11.25 & 14.2 & 25,100 & $5-10$ \\
\hline
\end{tabular}

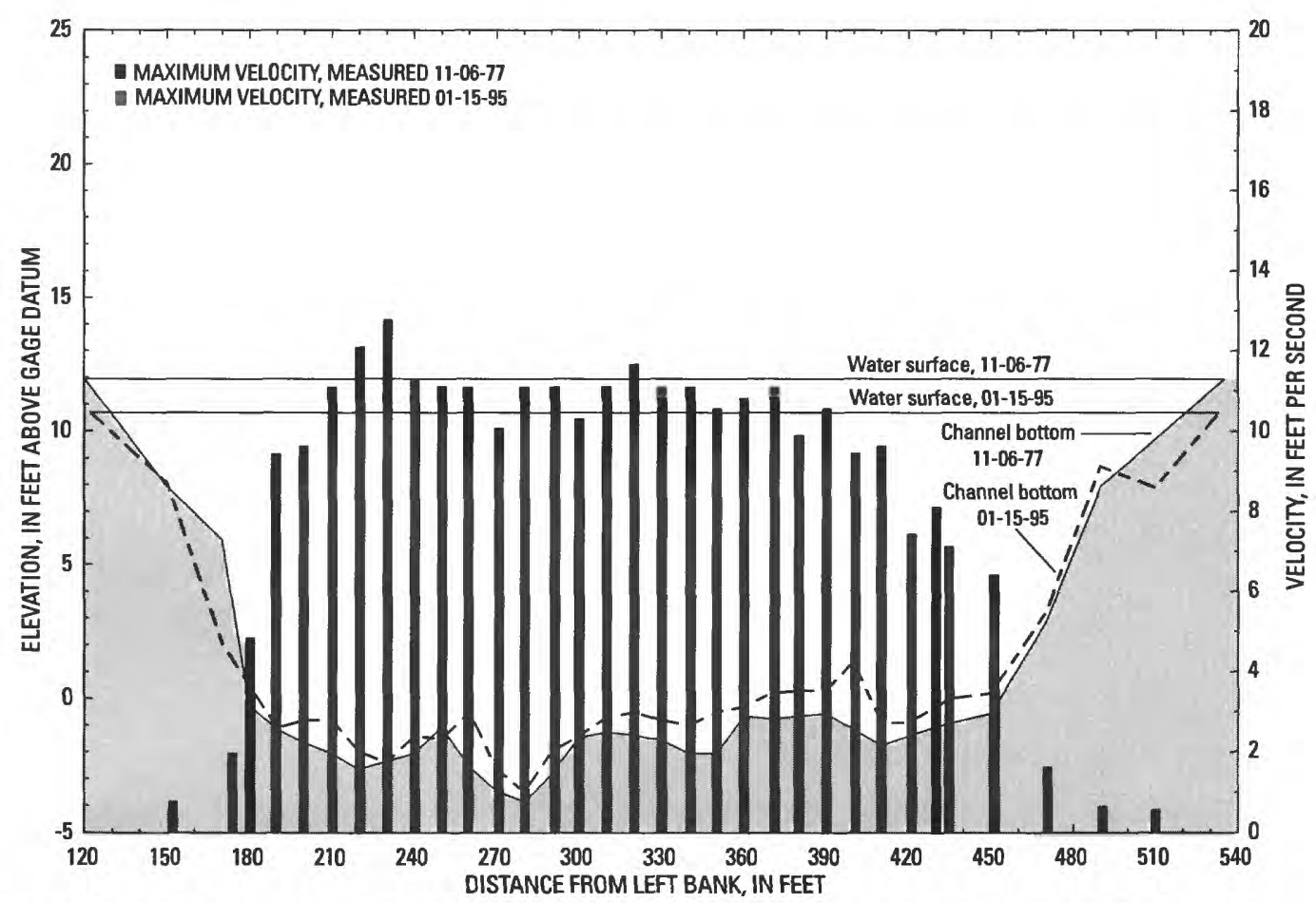

Figure 20. Channel cross sections and maximum flow velocities at station 03451500 French Broad River at Asheville, N.C. 
DISCHARGE MEASUREMENT DATA FOR STATION 03451500 FRENCH BROAD RIVER AT ASHEVILLE, N.C.

[ft, foot; ft/s, foot per second; nd, no data]

\begin{tabular}{|c|c|c|c|c|c|}
\hline \multicolumn{3}{|c|}{ Discharge measurement date, 11-06-77 } & \multicolumn{3}{|c|}{ Discharge measurement date, 01-15-95 } \\
\hline $\begin{array}{l}\text { Distance from } \\
\text { starting point on } \\
\text { left bank } \\
\text { (ft) }\end{array}$ & $\begin{array}{c}\text { Depth } \\
\text { (ft) }\end{array}$ & $\begin{array}{l}\text { Maximum } \\
\text { velocity } \\
\text { (ft/s) }\end{array}$ & $\begin{array}{l}\text { Distance from } \\
\text { starting point on } \\
\text { left bank } \\
\text { (ft) }\end{array}$ & $\begin{array}{c}\text { Depth } \\
\text { (ft) }\end{array}$ & $\begin{array}{l}\text { Maximum } \\
\text { veiocity } \\
\text { (ft/s) }\end{array}$ \\
\hline 120.00 & 0 & 0.00 & 122.00 & 0 & 0.00 \\
\hline 150.00 & 4 & 0.76 & 150.00 & 2.6 & 0.33 \\
\hline 180.00 & 12.2 & 4.83 & 190.00 & 11.8 & 6.29 \\
\hline 190.00 & 13.1 & 9.43 & 200.00 & 11.5 & 8.71 \\
\hline 200.00 & 13.6 & 9.63 & 210.00 & 11.5 & 9.89 \\
\hline 210.00 & 14 & 11.08 & 220.00 & 12.7 & 10.88 \\
\hline 250.00 & 13 & 11.08 & 260.00 & 11.2 & 9.07 \\
\hline 260.00 & 14.5 & 11.08 & 270.00 & 13.3 & 9.46 \\
\hline 270.00 & 15.4 & 10.07 & 280.00 & 14.2 & 10.36 \\
\hline 280.00 & 15.8 & 11.08 & 290.00 & 12.7 & 10.36 \\
\hline 290.00 & 14.8 & 11.09 & 300.00 & 12.1 & 9.46 \\
\hline 300.00 & 13.4 & 10.30 & 310.00 & 11.4 & 9.46 \\
\hline 310.00 & 13.2 & 11.08 & 320.00 & 11.2 & 9.89 \\
\hline 320.00 & 13.3 & 11.66 & 330.00 & 11.5 & 9.07 \\
\hline 330.00 & 13.5 & 11.08 & 340.00 & 11.7 & 8.71 \\
\hline 410.00 & 13.7 & 9.63 & 400.00 & 9.3 & 9.46 \\
\hline 430.00 & 13 & 8.11 & 410.00 & 11.6 & 8.71 \\
\hline 450.00 & 12.5 & 6.40 & 420.00 & 11.6 & 7.43 \\
\hline 470.00 & 9.1 & 1.60 & 435.00 & 10.7 & 7.11 \\
\hline 490.00 & 4 & 0.00 & 450.00 & 10.5 & 5.20 \\
\hline 535.00 & 0 & 0.00 & 470.00 & 7.5 & 1.44 \\
\hline nd & nd & nd & 490.00 & 2 & 0.64 \\
\hline nd & nd & nd & 510.00 & 2.8 & 0.56 \\
\hline nd & nd & nd & 533.00 & 0 & 0.00 \\
\hline
\end{tabular}




\section{SOUTH TOE RIVER NEAR CELO, N.C.}

GAGE LOCATION.--Lat 3549'53", long 82'11'04", Yancey County, Hydrologic Unit 06010108, on right bank on Secondary Road 1168, $800 \mathrm{ft}$ upstream from bridge on Secondary Road 1167, $0.3 \mathrm{mi}$ downstream of Whiteoak Creek, $1.9 \mathrm{mi}$ southeast of Celo, and at mile 20.1.

MEASUREMENT LOCATION.--Upstream side of bridge on Secondary Road $1167,800 \mathrm{ft}$ downstream of gage.

DRAINAGE AREA.--43.3 $\mathrm{mi}^{2}$.

PERIOD OF RECORD.--July 1957 to current year.

GAGE.--Water-stage recorder. Elevation of gage is 2,658 ft above sea level, from topographic map. Satellite telemetry at station.

DISCHARGE MEASUREMENT SUMMARY

[ft/s, foot per second; $\mathrm{ft}$, foot; $\mathrm{ft}^{3} / \mathrm{s}$, cubic foot per second]

\begin{tabular}{ccccccc}
\hline $\begin{array}{c}\text { Date } \\
\text { of } \\
\text { measurement }\end{array}$ & $\begin{array}{c}\text { Stream stage } \\
\text { (feet above } \\
\text { datum) }\end{array}$ & $\begin{array}{c}\text { Average } \\
\text { velocity } \\
(\mathrm{ft} / \mathrm{s})\end{array}$ & $\begin{array}{c}\text { Maximum } \\
\text { velocity } \\
(\mathrm{ft} / \mathrm{s})\end{array}$ & $\begin{array}{c}\text { Maximum } \\
\text { depth } \\
(\mathrm{ft})\end{array}$ & $\begin{array}{c}\text { Discharge } \\
\left(\mathrm{ft}^{3} / \mathrm{s}\right)\end{array}$ & $\begin{array}{c}\text { Recurrence } \\
\text { interval } \\
(\text { years) }\end{array}$ \\
\hline $01-14-95$ & 5.44 & 5.88 & 9.07 & 7.6 & 4,540 \\
\hline
\end{tabular}

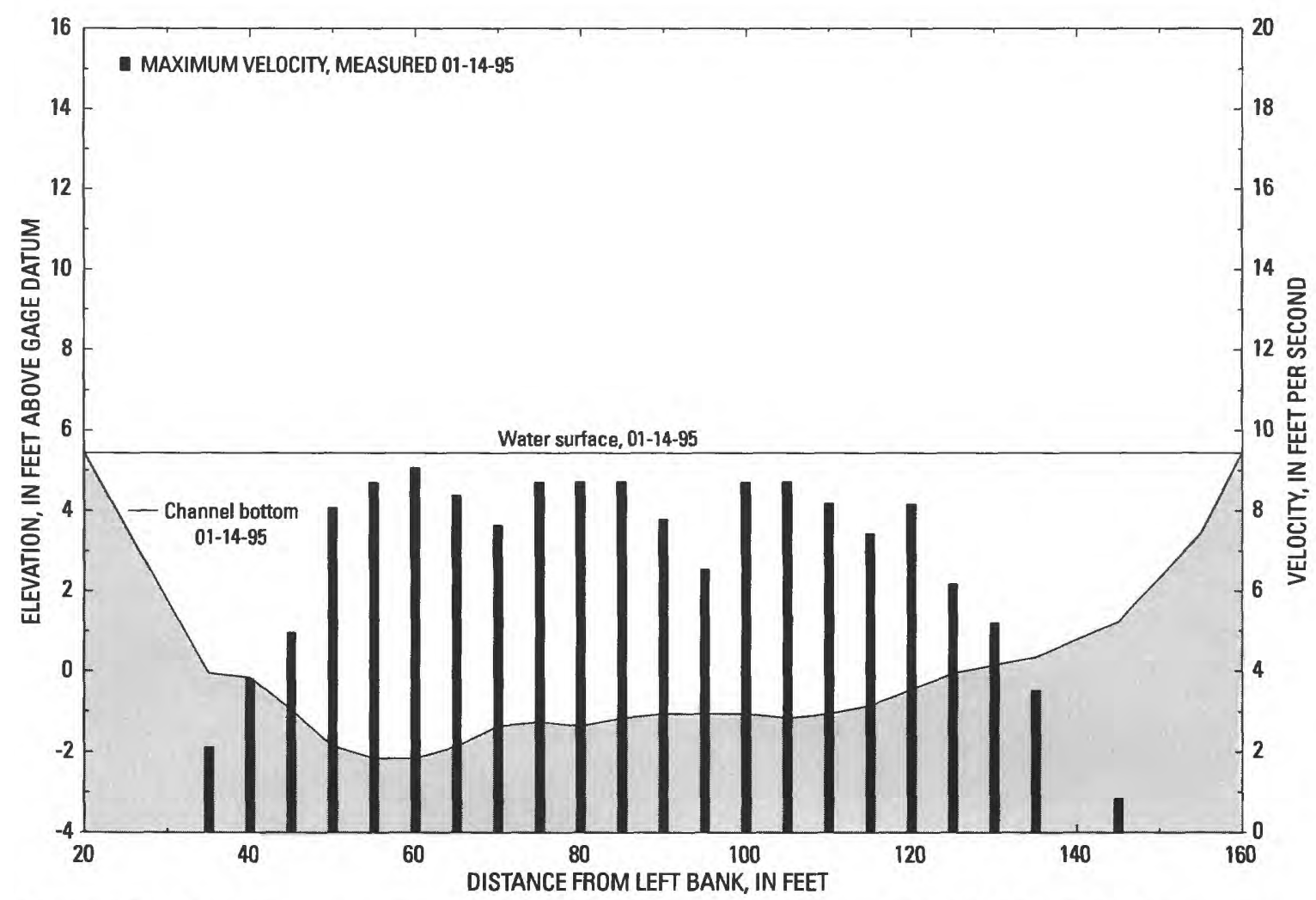

Figure 21. Channel cross section and maximum flow velocity at station 03463300 South Toe River near Celo, N.C. 
DISCHARGE MEASUREMENT DATA FOR STATION 03463300 SOUTH TOE RIVER NEAR CELO, N.C.

[ft, foot; ft/s, foot per second]

\begin{tabular}{|c|c|c|}
\hline \multicolumn{3}{|c|}{ Discharge measurement date, 01-14-95 } \\
\hline $\begin{array}{l}\text { Distance from } \\
\text { starting point on } \\
\text { left bank } \\
\text { (ft) }\end{array}$ & $\begin{array}{l}\text { Depth } \\
\text { (ft) }\end{array}$ & $\begin{array}{l}\text { Maximum } \\
\text { velocity } \\
\text { (ft/s) }\end{array}$ \\
\hline 20.00 & 0 & 0.00 \\
\hline 35.00 & 5.5 & 2.10 \\
\hline 40.00 & 5.6 & 3.80 \\
\hline 45.00 & 6.4 & 4.96 \\
\hline 50.00 & 7.3 & 8.07 \\
\hline 55.00 & 7.6 & 8.71 \\
\hline 60.00 & 7.6 & 9.07 \\
\hline 65.00 & 7.3 & 8.38 \\
\hline 70.00 & 6.8 & 7.64 \\
\hline 75.00 & 6.7 & 8.71 \\
\hline 80.00 & 6.8 & 8.71 \\
\hline 85.00 & 6.6 & 8.71 \\
\hline 90.00 & 6.5 & 7.78 \\
\hline 95.00 & 6.5 & 6.54 \\
\hline 100.00 & 6.5 & 8.71 \\
\hline 105.00 & 6.6 & 8.71 \\
\hline 110.00 & 6.5 & 8.17 \\
\hline 115.00 & 6.3 & 7.43 \\
\hline 120.00 & 5.9 & 8.17 \\
\hline 125.00 & 5.5 & 6.17 \\
\hline 130.00 & 5.3 & 5.20 \\
\hline 135.00 & 5.1 & 3.50 \\
\hline 145.00 & 4.2 & 0.84 \\
\hline 155.00 & 2 & 0.00 \\
\hline 160.00 & 0 & 0.00 \\
\hline
\end{tabular}




\section{CARTOOGECHAYE CREEK NEAR FRANKLIN, N.C.}

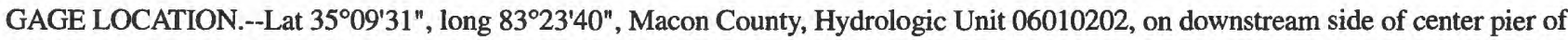
bridge on Secondary Road 1152, 0.1 mi downstream of unnamed creek, $1.8 \mathrm{mi}$ south of Franklin, and 1.9 mi upstream from mouth.

MEASUREMENT LOCATION.--Upstream side of bridge on Secondary Road 1152.

DRAINAGE AREA.--57.1 $\mathrm{mi}^{2}$.

PERIOD OF RECORD.--Occasional low-flow measurements, water years 1944, 1947, 1953-55, 1958, 1960. June 1961 to current year.

GAGE.--Water-stage recorder. Datum of gage is $2,017.18 \mathrm{ft}$ above sea level. Satellite telemetry at station.

EXTREMES OUTSIDE PERIOD OF RECORD.--Flood in June 1949 reached a stage of $15.6 \mathrm{ft}$, from studies by Tennessee Valley Authority; discharge, about $7,000 \mathrm{ft}^{3} / \mathrm{s}$.

\section{DISCHARGE MEASUREMENT SUMMARY}

[ft/s, foot per second; $\mathrm{ft}$, foot; $\mathrm{ft}^{3} / \mathrm{s}$, cubic foot per second]

\begin{tabular}{ccccccc}
\hline $\begin{array}{c}\text { Date } \\
\text { of } \\
\text { measurement }\end{array}$ & $\begin{array}{c}\text { Stream stage } \\
\text { (feet above } \\
\text { datum) }\end{array}$ & $\begin{array}{c}\text { Average } \\
\text { velocity } \\
(\mathrm{ft} / \mathrm{s})\end{array}$ & $\begin{array}{c}\text { Maximum } \\
\text { velocity } \\
(\mathrm{ft} / \mathrm{s})\end{array}$ & $\begin{array}{c}\text { Maximum } \\
\text { depth } \\
(\mathrm{ft})\end{array}$ & $\begin{array}{c}\text { Discharge } \\
\left(\mathrm{ft}^{3} / \mathrm{s}\right)\end{array}$ & $\begin{array}{c}\text { Recurrence } \\
\text { interval } \\
(\text { years) }\end{array}$ \\
\hline $02-16-95$ & 12.16 & 4.09 & 7.70 & 12.5 & 3,220 \\
\hline
\end{tabular}

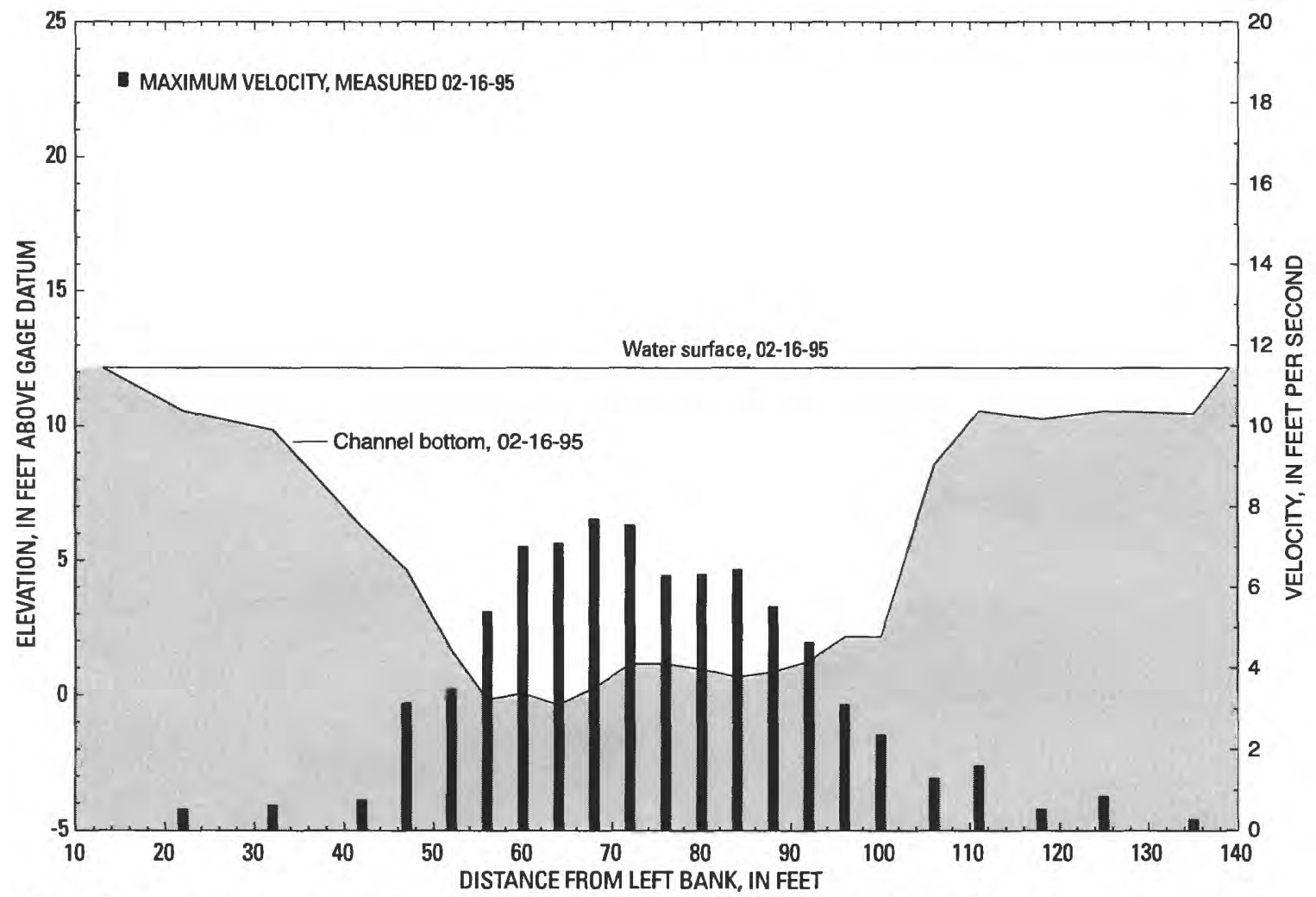

Figure 22. Channel cross section and maximum flow velocity at station 03500240 Cartoogechaye Creek near Franklin, N.C. 
DISCHARGE MEASUREMENT DATA FOR STATION 03500240 CARTOOGECHAYE CREEK NEAR FRANKLIN, N.C.

[ft, foot; ft/s, foot per second; nd, no data]

\begin{tabular}{ccc}
\hline \multicolumn{2}{c}{ Discharge measurement date, 02-16-95 } \\
\hline $\begin{array}{c}\text { Distance from } \\
\text { starting point on } \\
\text { left bank } \\
\text { (ft) }\end{array}$ & $\begin{array}{c}\text { Depth } \\
\text { (ft) }\end{array}$ & $\begin{array}{c}\text { Maximum } \\
\text { velocity } \\
\text { (fts) }\end{array}$ \\
\hline 13.00 & 0 & 0.00 \\
22.00 & 1.6 & 0.52 \\
32.00 & 2.3 & 0.61 \\
42.00 & 5.9 & 0.75 \\
47.00 & 7.5 & 3.14 \\
52.00 & 10.5 & 3.48 \\
56.00 & 12.3 & 5.39 \\
60.00 & 12.1 & 7.01 \\
64.00 & 12.5 & 7.10 \\
68.00 & 11.9 & 7.70 \\
72.00 & 11 & 7.55 \\
76.00 & 11 & 6.29 \\
80.00 & 11.2 & 6.33 \\
84.00 & 11.5 & 6.44 \\
88.00 & 11.3 & 5.52 \\
92.00 & 10.9 & 4.63 \\
96.00 & 10 & 3.10 \\
100.00 & 10 & 2.36 \\
106.00 & 3.6 & 1.28 \\
111.00 & 1.6 & 1.60 \\
118.00 & 1.9 & 0.52 \\
125.00 & 1.6 & 0.84 \\
135.00 & 1.7 & 0.27 \\
139.00 & 0 & 0.00 \\
\hline & & \\
\hline
\end{tabular}

